\title{
Climate change signal in global agriculture emerges earlier in new generation of climate and crop models
}

Jonas Jaegermeyr ( $\square$ jonas.jaegermeyr@columbia.edu )

Columbia University https://orcid.org/0000-0002-8368-0018

Christoph Müller

Potsdam Institute for Climate Impact Research https://orcid.org/0000-0002-9491-3550

Alex Ruane

NASA Goddard Institute for Space Studies https://orcid.org/0000-0002-5582-9217

Joshua Elliott

Columbia University

Juraj Balkovic

International Institute for Applied Systems Analysis (IIASA)

Oscar Castillo

University of Florida

\section{Babacar Faye}

Institut de recherche pour le développement

lan Foster

University of Chicago

Christian Folberth

International Institute for Applied Systems Analysis https://orcid.org/0000-0002-6738-5238

James Franke

University of Chicago

Kathrin Fuchs

Karlsruhe Institute of Technology

Jose Guarin

Columbia University https://orcid.org/0000-0002-3167-4329

Jens Heinke

Potsdam Institute for Climate Impacts Research

\section{Gerrit Hoogenboom}

University of Florida https://orcid.org/0000-0002-1555-0537

\section{Toshichika lizumi}

National Agriculture and Food Research Organization https://orcid.org/0000-0002-0611-4637

Atul Jain 
University of Illinois at Urbana-Champaign https://orcid.org/0000-0002-4051-3228

\section{David Kelly}

University of Chicago

\section{Nikolay Khabarov}

International Institute for Applied Systems Analysis https://orcid.org/0000-0001-5372-4668

\section{Stefan Lange}

Potsdam Institute for Climate Impact Research (PIK), Member of the Leibniz Association https://orcid.org/0000-0003-2102-8873

\section{Tzu-Shun Lin}

University of Illinois at Urbana-Champaign

\section{Wenfeng Liu}

China Agricultural University

\section{Oleksandr Mialyk}

University of Twente https://orcid.org/0000-0002-7495-2325

\section{Sara Minoli}

PIK

\section{Elisabeth Moyer}

University of Chicago https://orcid.org/0000-0003-1829-5196

\section{Masashi Okada}

National Institute for Environmental Studies

\section{Meridel Phillips}

Columbia University

\section{Cheryl Porter}

University of Florida https://orcid.org/0000-0001-7269-6543

\section{Sam Rabin}

Karlsruhe Institute of Technology

\section{Clemens Scheer}

Karlsruhe Institute of Technology

\section{Julia Schneider}

Ludwig-Maximilians-Universität München

\section{Joep Schyns}

University of Twente https://orcid.org/0000-0001-5058-353X

\section{Rastislav Skalský}

International Institute for Applied Systems Analysis https://orcid.org/0000-0002-0983-6897

\section{Andrew Smerald}

Karlsruhe Institute of Technology

\section{Tommaso Stella}

Leibniz Centre for Agricultural Landscape Research https://orcid.org/0000-0002-3018-6585

\section{Haynes Stephens}


University of Chicago https://orcid.org/0000-0002-2258-5244

\section{Heidi Webber}

Leibniz Centre for Agricultural Landscape Research https://orcid.org/0000-0001-8301-5424

\section{Florian Zabel}

Ludwig-Maximilians-Universität München https://orcid.org/0000-0002-2923-4412

\section{Cynthia Rosenzweig}

NASA Goddard Institute for Space Studies https://orcid.org/0000-0002-8541-2201

\section{Article}

Keywords: crop model, climate-related impacts, yield responses

Posted Date: August 12th, 2021

DOI: https://doi.org/10.21203/rs.3.rs-101657/v1

License: (c) (i) This work is licensed under a Creative Commons Attribution 4.0 International License. Read Full License

Version of Record: A version of this preprint was published at Nature Food on November 1st, 2021. See the published version at https://doi.org/10.1038/s43016-021-00400-y. 
Climate change signal in global agriculture emerges earlier in new generation of climate and crop models

Jonas Jägermeyr ${ }^{1,2,3}$, Christoph Müller ${ }^{3}$, Alex C. Ruane ${ }^{1}$, Joshua Elliott ${ }^{4}$, Juraj Balkovic ${ }^{5,19}$, Oscar Castillo ${ }^{6}$, Babacar Faye ${ }^{7}$, lan Foster ${ }^{8}$, Christian Folberth ${ }^{5}$, James A. Franke ${ }^{9,4}$, Kathrin Fuchs $^{10}$, Jose Guarin ${ }^{1,2}$, Jens Heinke ${ }^{3}$, Gerrit Hoogenboom ${ }^{6,11}$, Toshichika lizumi ${ }^{12}$, Atul K. Jain $^{13}$, David Kelly ${ }^{8}$, Nikolay Khabarov ${ }^{5}$, Stefan Lange ${ }^{3}$, Tzu-Shun Lin ${ }^{13}$, Wenfeng Liu ${ }^{14}$, Oleksandr Mialyk ${ }^{15}$, Sara Minoli ${ }^{3}$, Elisabeth J. Moyer ${ }^{9,4}$, Masashi Okada ${ }^{16}$, Meridel Phillips ${ }^{1,2}$, Cheryl Porter ${ }^{6}$, Sam Rabin ${ }^{10}$, Clemens Scheer ${ }^{10}$, Julia M. Schneider ${ }^{17}$, Joep F. Schyns ${ }^{15}$, Rastislav Skalsky ${ }^{5,20}$, Andrew Smerald ${ }^{10}$, Tommaso Stella ${ }^{18}$, Haynes Stephens ${ }^{9}$, Heidi Webber $^{18}$, Florian Zabel ${ }^{17}$, Cynthia Rosenzweig ${ }^{1}$

${ }^{1}$ NASA Goddard Institute for Space Studies, New York, USA

${ }^{2}$ Columbia University, Center for Climate Systems Research, New York, USA

${ }^{3}$ Potsdam Institute for Climate Impacts Research (PIK), Member of the Leibniz Association, Potsdam, Germany

${ }^{4}$ Center for Robust Decision-making on Climate and Energy Policy (RDCEP), University of Chicago, Chicago, USA

5 International Institute for Applied Systems Analysis, Laxenburg, Austria

${ }^{6}$ Agricultural \& Biological Engineering Department, University of Florida, Gainesville, Florida, USA

${ }^{7}$ Institut de recherche pour le développement (IRD) ESPACE-DEV, Montpellier, France

${ }^{8}$ Department of Computer Science, University of Chicago, Chicago, Illinois, USA

${ }^{9}$ Department of the Geophysical Sciences, University of Chicago, Chicago, Illinois, USA

${ }^{10}$ Institute of Meteorology and Climate Research, Atmospheric Environmental Research, Karlsruhe Institute of Technology, Garmisch-Partenkirchen, Germany

${ }^{11}$ Institute for Sustainable Food Systems, University of Florida, Gainesville, Florida, USA

${ }^{12}$ Institute for Agro-Environmental Sciences, National Agriculture and Food Research Organization, Tsukuba, Japan

${ }^{13}$ Department of Atmospheric Sciences, University of Illinois, Urbana, Illinois, USA

${ }^{14}$ Center for Agricultural Water Research in China, College of Water Resources and Civil Engineering, China Agricultural University, Beijing, China

${ }^{15}$ Multidisciplinary Water Management group, University of Twente, Enschede, The Netherlands

${ }^{16}$ Center for Social and Environmental Systems Research, National Institute for Environmental Studies, Tsukuba, Japan

${ }^{17}$ Ludwig-Maximilians-Universität München (LMU), Munich, Germany

${ }^{18}$ Leibniz Centre for Agricultural Landscape Research (ZALF), Müncheberg, Germany

${ }^{19}$ Faculty of Natural Sciences, Comenius University in Bratislava, Bratislava, Slovak Republic

${ }^{20}$ Soil Science and Conservation Research Institute, National Agricultural and Food Centre, Bratislava, Slovak Republic

Potential climate-related impacts on future crop yield are a major societal concern first surveyed in a harmonized multi-model effort in 2014 . We report here on new $21^{\text {st }}$-century projections using ensembles of latest-generation crop and climate models. Results suggest markedly more pessimistic yield responses for maize, soybean, and rice compared to the original ensemble. Mean end-of-century maize productivity is shifted from +5 to $-6 \%$ (SSP126) and +1 to $-24 \%$ (SSP585) - explained by warmer climate projections and improved crop model sensitivities. In contrast, wheat shows stronger gains ( +9 shifted to $+18 \%$, SSP585), linked to higher $\mathrm{CO}_{2}$ concentrations and expanded 
high-latitude gains. The 'emergence' of climate impacts - when the change signal emerges from the noise - consistently occurs earlier in the new projections for several main producing regions before 2040 . While future yield estimates remain uncertain, these results suggest that major breadbasket regions will face distinct anthropogenic climatic risks sooner than previously anticipated.

Climate change already affects agricultural productivity worldwide via many mechanisms, driven largely by warmer mean and extreme temperatures, altered precipitation regimes and drought patterns, and elevated atmospheric $\mathrm{CO}_{2}$ concentrations $\left(\left[\mathrm{CO}_{2}\right]\right)^{1}$. Uncertainties arising from greenhouse gas emission scenarios, climate model projections, and the understanding and representation of complex impact processes render estimates of future crop yield highly uncertain $^{2}$. A way towards improving yield projections is the development of benchmarked multimodel ensemble simulations driven by harmonized simulation protocols ${ }^{3}$. Facilitated by the Agricultural Model Intercomparison and Improvement Project (AgMIP) ${ }^{4}$ and the Inter-Sectoral

61 Impact Model Intercomparison Project (ISIMIP) ${ }^{5}$, here we present a new systematic assessment of agricultural yield projections, based on a protocol similar to the one used by Coupled Model Intercomparison Project (CMIP) for climate models ${ }^{6}$. Previous projections of AgMIP's Global Gridded Crop Model Intercomparison (GGCMI) based on CMIP5 identified substantial climate impacts on all major crops, with strong temperature and $\mathrm{CO}_{2}$ responses and regional patterns of losses and gains ${ }^{7}$. As the first systematic intercomparison, GGCMI-CMIP5 (hereafter 'GC5') demonstrated in 2014 that crop models might indeed introduce larger uncertainty than current climate models. CMIP6 now provides new reference climate model projections ${ }^{8,9}$, and recently improved bias-adjustment and downscaling methods ${ }^{10}$ benefit the impact modeling community and support an advanced ensemble of process-based crop models. With improved and further

71 harmonized inputs and configuration of cropping systems, GGCMI is able to provide a new

72 standard in crop yield projections for the $21^{\text {st }}$ century for several major crops using state-of-the- 
75 Climate change impacts are often quantified in terms of differences over time, but especially in

76 view of adaptation measures, it is the amplitude of the change compared to the local

77 background variability and uncertainty of the recent past that is often more relevant ${ }^{11}$. Time of

78 climate impact emergence (TCIE) — the point in time by which the yield levels of exceptional

79 years (negative or positive) have become the new norm - is a critical measure for risk

80 assessment. Time of emergence ${ }^{12}$ metrics have been applied to climate variables including

81 temperature ${ }^{13,14}$, precipitation ${ }^{15}$, and others ${ }^{16,17}$ and demonstrate that major food producing

82 regions are increasingly facing changing climate profiles in the near term. Here we introduce the

83 TCIE concept with respect to future agricultural risks.

85 The analyses presented here shed new light on the projected effects of elevated $\left[\mathrm{CO}_{2}\right]$, which

86 have been neglected in many previous studies that focused on direct temperature responses ${ }^{18-}$

$87{ }^{20} . \mathrm{CO}_{2}$ effects are among the largest sources of uncertainty inflating the range of crop model

88 projections by the end of the century ${ }^{21-24}$, but they must be reflected in plausible future yield

89 projections ${ }^{25}$. The uncertainty in the mechanisms and overall size of the effects of $\mathrm{CO}_{2}$

90 fertilization manifested in farmers' fields are reflected in a wide range of $\mathrm{CO}_{2}$ sensitivities among

91 the crop models contributing to the GGCMI archive 21,25 .

92

93 Here we present an ensemble of process-based projections of global productivity estimates for

94 the major crops for the $21^{\text {st }}$ century. This work represents the first update since GC5 in $2014^{7}$

95 and includes updated climate projections based on CMIP6 and latest-generation crop models

96 for maize, wheat, rice, and soybean. This study is based on constant management

97 assumptions, focusing on the isolated climate change effect on current crop production

98 systems. Opportunities associated with farming system adaptation and management trends will

99 be addressed in upcoming GGCMI simulations. 
101 The simulation protocol is based on two Shared Socioeconomic Pathways related to

102 Representative Concentration Pathways (RCPs), RCP2.6 and RCP8.5 (hereafter 'SSP126' and

103 'SSP585'; adaptation measures associated with the SSPs are not considered) ${ }^{9}$, chosen to

104 sample the range of available scenarios ${ }^{26}$ and to make the results comparable with GC5.

105 Twelve GGCMs each simulated 5 GCM forcings, resulting in nearly 240 climate-crop model

106 realizations per crop (GGCMs $\times$ GCMs $\times$ RCPs $\times \mathrm{CO}_{2}$ settings). The climate projections from the

1075 GCMs (Table S1), centrally bias-adjusted and downscaled for different research sectors, were

108 selected by ISIMIP based on benchmark performance, equilibrium climate sensitivity, and

109 output availability (see Methods). All simulations were carried out globally on a $0.5^{\circ}$ grid,

110 covering the time period 1850 to 2100 . We evaluate results based on the transient atmospheric

$111 \mathrm{CO}_{2}$ concentration (i.e., 'default' $\left[\mathrm{CO}_{2}\right]$ ) and only refer to counterfactual simulations without

$112\left[\mathrm{CO}_{2}\right]$ increase after the year 2015 ('constant' $\left[\mathrm{CO}_{2}\right]$ ) to quantify the $\mathrm{CO}_{2}$ fertilization effect for

113 further uncertainty evaluation and climate change factor attribution.

114

115 Recent literature has focused on capturing the temperature sensitivity of crops ${ }^{18-20,27-29}$ in

116 isolation. To quantify climate change impacts more comprehensively, additional factors

117 including precipitation changes, temperature-moisture feedbacks, and $\left[\mathrm{CO}_{2}\right]$ need to be

118 considered. The aims of this first GC6 study are to: i) provide new ensemble projections for the

119 productivity of major crops under climate change, ii) assess climate change impacts on crop

120 yields from a risk perspective, employing the TCIE concept, iii) improve understanding of

121 regional patterns of change, and iv) explore drivers of uncertainty related to climate models,

122 crop models, and responses to $\left[\mathrm{CO}_{2}\right]$.

\section{Global production response of major crops}

124 The ensemble response across the new generation of climate and crop models to the SSP126

125 and SSP585 forcing is markedly more pronounced than in $\mathrm{GC}^{7}$ (Fig. 1). Wheat results are 
126 more optimistic, while maize, soybean, and rice results are decisively more pessimistic. For

127 maize, the most important global crop in terms of total production and food security in many

128 regions, the mean end-of-century (2069-2099) global productivity response is $\sim 10 \%$ (SSP126)

129 and $\sim 20 \%$ (SSP585) lower than in GC5. This shifts the SSP585 estimate from $+1 \%$

130 (interquartile range of crop-climate model combinations: -10 to $+8 \%$ ) to $-24 \%$ (-38 to $-7 \%$ ) and

131 for SSP126 from +5 to $-6 \%$. For wheat, the second largest global crop in terms of production,

132 the SSP585 ensemble estimate is shifted upwards from $+10 \%(-1$ to $+15 \%)$ to $+18 \%$ ( -2 to

$133+39 \%$ ), and under SSP126 from +5 to $+9 \%$. The SSP585 ensemble estimates for soybean are

134 revised downward from $+15 \%(-8$ to $+36 \%)$ to $-2 \%(-21$ to $+17 \%)$ and for rice from $+23 \%(+1$ to

$135+33 \%)$ to $+2 \%(-15$ to $+12 \%)$. Overall, the new climate and crop model combinations narrow the

136 range of crop yield projections for soybean and rice, but disagreement among crop models

137 remains substantial and is largely indecisive about the sign of change at the global level (p-

138 value $>0.5$ for both crops). The maize and wheat responses are robust and became more

139 distinct since GC5. While the range of crop projections somewhat increased, $85 \%$ of model

140 combinations indicate negative maize changes and $73 \%$ project positive wheat changes under

141 SSP585. Both responses are now statistically significant ( $p$-value $<10^{-5}$ ); the maize response in

142 GC5 was not ( $p$-value > 0.6). There is larger agreement on positive change for wheat under

143 SSP126 (89\%) than under SSP585, indicating peak-and-decline trajectories for parts of the

144 ensemble under high-emissions scenarios (Fig. S1).

145

146 As a $\mathrm{C}_{4}$ crop, maize has a smaller capacity to benefit from elevated $\left[\mathrm{CO}_{2}\right]^{30}$, and it is also grown

147 across a wider range of low latitudes that are projected to experience the largest adverse

148 impacts due in large part to current proximity to crop-limiting temperature thresholds ${ }^{31}$. As a $\mathrm{C}_{3}$

149 crop, the positive wheat response is explained by its relatively stronger $\mathrm{CO}_{2}$ response and the

150 fact that global warming leads to wheat yield increases in high-latitude regions that are currently

151 temperature-limited ${ }^{29}$. 
153 Three factors explain the more-pronounced crop yield response in GC6. First, CMIP6 has

154 markedly higher $\left[\mathrm{CO}_{2}\right]$ than CMIP5 (Fig. 2), with year 2099 concentrations increased from 927

$155 \mathrm{ppm}$ (RCP8.5) to $1122 \mathrm{ppm}(\mathrm{SSP} 585)^{9}$. Second, CMIP6 has a higher average end-of-century

156 warming level compared to CMIP5, adequately represented in the 5 GCMs sampled here (Table

157 S1, S2). While both RCP2.6 and RCP8.5 are on average $\sim 0.3^{\circ} \mathrm{C}$ warmer in CMIP6 than CMIP5

158 over land and oceans, the difference is even more pronounced $\left(>0.5^{\circ} \mathrm{C}\right)$ across main maize-

159 producing regions (Fig. 2). Third, the new crop model ensemble features advanced versions of

160 previous models, several new members, and improved input data, which resulted in more

161 realistic sensitivities to climate and $\left[\mathrm{CO}_{2}\right]$ changes (see details below).

162 Emergence of the climate change signal in agriculture

163 The Time of Climate Impact Emergence (TCIE) describes the point in time when average

164 climate change impacts are projected to occur outside the envelope of historical variability and

165 uncertainty ('noise'). We define TCIE as the year in which the multi-model 25yr moving-average

166 crop production change ('signal') emerges from the noise (i.e., standard deviation of simulated

167 variability across all GCM x GGCM combinations in 1983-2013).

Maize consistently shows emerging negative productivity changes ('negative TCIE') among

170 major producer regions. The ensemble median signal emerges from the noise at global level in

171 the year 2032 under SSP585 and the year 2051 under SSP126 (Fig. 3). Of all individual GCM x

172 GGCM realizations, 84\% show a negative TCIE by 2099 under SSP585 (52\% under SSP126)

173 and the inter-quartile range spans from 2014 to 2056, indicating sizeable agreement among

174 models. This is a substantial shift away from the GC5 simulations in which the ensemble

175 median shows no emergence by 2099 under any emission pathway, only seen in $46 \%$ of

176 individual GCM x GGCM combinations under RCP8.5 (inter-quartile range 2044-2080). Overall, 
177 the TCIE signal at global level is shifted $30-40$ years earlier and is more pronounced in the new 178 generation of climate and crop model projections (Fig. 4).

By the end of the century, 10\% (SSP126) to 74\% (SSP585) of current global maize cultivation areas are projected to undergo negative TCIE (Fig. 5). Under SSP585 this trajectory is markedly earlier, with higher late-century fractions of cropland area affected compared to the respective 47\% in GC5 (RCP8.5). Crop models indicate early negative maize TCIE before 2040 even under SSP126 in Central Asia, the Middle East, Southern Europe, Western USA, and tropical South America. Projections referencing the 1983-2013 period suggest that the mean yield signal is already starting to emerge in some of these regions (Fig. 3e and Fig. 5), patterns largely in line with recent observations ${ }^{15,32,33}$. The tropical zone is the only climate zone in which the GC5 ensemble median also indicated a negative maize TCIE (Fig. 3e).

The standard deviation of grid-level TCIE estimates under SSP585 ranges between 25 and 35 years across most breadbasket regions, with slightly higher values under SSP126 (Fig. S2). Such uncertainty ranges are in line with time of emergence estimates for climatological variables, yet somewhat higher due to the additional layer of crop model uncertainties ${ }^{12,13}$.

194 Clearest emergence signals, i.e., largest signal-to-noise ratios with values $<-2$, are found 195 among lower latitudes in the tropics but also in Central Asia, the Middle East, and Western USA 196 (Fig. S3). As internal variability — and thus total noise — decreases with averaging, earlier 197 TCIE is generally found for larger spatial scales.

199 For wheat, ensemble projections indicate TCIE of positive productivity changes ('positive TCIE') 200 at the global level (Fig. 3b) and across large parts of currently cultivated areas (Fig. 5). While 201 also found in GC5 simulations, TCIE is shifted $\sim 10$ years earlier in GC6, suggesting that 202 climate-related increases might occur globally within the next few years (year 2023 under 
SSP585, year 2025 under SSP126; inter-quartile ranges 2014-2029 and 2015-2029) and across

204 major breadbasket regions within the next two decades (Fig. 5). In some regions we already

205 detect a TCIE signal today, which is in line with the range of time of emergence estimates for

206 temperature and precipitation ${ }^{13,15}$. Such effects are difficult to distinguish from rapidly changing

207 management practices in observational data, but climate change impacts have been

208 documented for example in Central and South Asia, Northern China, and the USA ${ }^{32,34}$. The

209 TCIE estimates for wheat show high consistencies across the model ensemble $-76 \%$

210 (SSP126) and 88\% (SSP585) of individual model combinations show positive TCIE by 2099. As

211 for maize, the TCIE signal is shifted earlier and is more pronounced in GC6 than in GC5 (Fig.

$2124)$

213

214 The share of wheat cultivation areas projected to see positive TCIE increased substantially in

215 GC6, from 8\% (GC5, RCP8.5) to 37\% (GC6, SSP585; Fig. 5f). This share levels off by mid-

216 century, a result of peak-and-decline trajectories seen in some crop models (Fig. 5f ; compare

217 Fig. 3d and Fig. S3 for regions that show TCIE early on but not by late century). Wheat also

218 exhibits negative TCIE among important growing regions in South Asia, Southern USA, Mexico,

219 and parts of South America around mid-century. The uncertainty among grid-level TCIE

220 estimates is generally higher for wheat than for maize (Fig. S2) and the extent of areas with very

221 high signal-to-noise ratios (i.e., >2) is smaller (Fig. S3).

223 Ensemble median soybean and rice productivity peak mid-century and decline towards the end 224 of the century at the global level (Fig. S4). The soybean response exhibits late-century negative 225 TCIE (year 2096) under SSP585; rice on the other hand shows early positive TCIE (year 2030, 226 SSP585) but late-century declines are not projected to reach the level of negative TCIE at the 227 global level. Rice is the only crop in this study that indicates positive TCIE in the tropics, which 
228 drives early net global gains before productivity is simulated to decline again by about 2060 229 (Fig. S4c).

\section{Regional patterns of yield change}

231 Projections of crop yield changes include regions of losses and gains for all crops (Fig. 3, S4).

232 Global average responses can hide important regional changes, which are supported by strong

233 crop model agreement. Maize projections show spatially homogeneous losses especially

234 among main growing regions in North America, Mexico, West Africa, Central Asia, and China, 235 where crop model agreement is high (Fig. 3c). The high-latitude gains found in GC5 are not as 236 prevalent in GC6 and associated with high crop model uncertainty and low baseline yields.

237 Wheat shows distinct geographic gradients with losses in spring wheat regions in Mexico, 238 Southern USA, South America, and South Asia, supported by good model agreement. Sizable 239 wheat gains are projected by many models for the North China Plains, Australia, Central Asia, 240 Middle East, and for the winter wheat growing regions in the Northern USA and Canada (Fig.

$2413 d$ ). Soybean shows the greatest losses in the main-producer regions - the USA, Brazil, and

242 Southeast Asia - paired with large gains across parts of China and generally higher latitudes

243 (Fig. S4). Major declines in rice yields are simulated in Central Asia, and gains in South Asia,

244 NE China, and South America. Both soybean and rice yield changes must be interpreted in view 245 of the wide range in crop model ensemble results (Fig. 1, S4). A breakdown of yield responses 246 for the top-10 producer countries per crop highlights a wide range of $\mathrm{CO}_{2}$ effects embedded in 247 the signal (Fig. S5, S6).

249 A latitudinal profile of yield changes under SSP585 - simulated in all grid cells irrespective of 250 the current cropland distribution - indicates that losses are most prevalent among low-latitude 251 tropical regions with highest gains found at higher latitudes beyond $50^{\circ} \mathrm{N}$ and $30^{\circ} \mathrm{S}$ for all crops 252 (Fig. 6). Maize exhibits widespread losses between $50^{\circ} \mathrm{N}$ and $30^{\circ} \mathrm{S}$, while losses for the other 
crops are more concentrated in the tropics with a less distinct signal for soybean and rice. Major

254 wheat breadbaskets are generally located at higher latitudes than maize, which further

255 contributes to overall wheat gains when aggregated across currently cultivated areas. Although

256 more than $90 \%$ of maize and wheat is currently produced in the temperate and subtropical

257 climate zones, major yield losses will affect the livelihoods and food security of many

258 smallholder farmers in the tropics. Overall, our results show that lower latitudes face the largest

259 losses for all crops, while higher latitudes see potential gains. These conclusions are in line with

260 the IPCC $\mathrm{AR}^{35}$ and recent studies ${ }^{7,36,37}$ and such uneven distribution of impacts may further

261 increase regional disparities that are a 'Reason for Concern'38 regarding climate change risks.

\section{Drivers of more pronounced ensemble response}

263 It is difficult to determine to what degree the differences in crop yield projections between GC6

264 and GC5 can be explained by the new atmospheric forcing, the new crop model ensemble, or

265 new input data. A subset of GC6 and GC5 crop models that participated in both ensembles

266 (albeit in different versions) shows very similar responses compared with the respective full

267 ensemble, suggesting that the crop model selection does not explain the differences (Fig. 7).

268 Further, standardized comparisons of crop model responses to specific mean temperature

269 increases over cropland areas ('warming sensitivity'; under constant $\left[\mathrm{CO}_{2}\right]$ conditions, but

270 including changes in other climate variables) from $1-2^{\circ} \mathrm{C}$ and from $2-3^{\circ} \mathrm{C}$, respectively, highlights

271 that the isolated warming sensitivity in GC6 has substantially increased for maize (from 2-3\% in

272 GC5 to 8-9\% in GC6) and decreased for wheat (from 7\% to 3-6\%; Fig. 7). With higher overall

273 warming levels in CMIP6, net warming-related maize losses by 2069-2099 thus increased from

$27412 \%\left(4.6^{\circ} \mathrm{C}\right.$ maize cropland warming) to $30 \%\left(5^{\circ} \mathrm{C}\right.$ maize cropland warming $)$ in GC6. Further,

275 the $\mathrm{CO}_{2}$ sensitivity at 500 and 700 ppm, but also net effects by the end of the century, have

276 decreased for both maize and wheat. In summary, the more pessimistic maize response in GC6

277 can largely be attributed to a higher sensitivity to warming and a lower compensating effect due 
278 to $\mathrm{CO}_{2}$ fertilization in the crop models, and to a smaller extent to the higher absolute warming

279 levels in CMIP6. For wheat on the other hand, the more optimistic response in GC6 can be

280 explained by lower losses per degree warming (with stronger temperature-related gains in high-

281 latitude regions), overcompensating for a lower $\mathrm{CO}_{2}$ fertilization effect than in $\mathrm{GC} 5$ (despite

282 higher total $\left[\mathrm{CO}_{2}\right]$ levels). For soybean and rice, in contrast, the more pessimistic response in

283 GC6 is largely attributed to higher warming levels in CMIP6 compounded by a higher crop

284 model sensitivity to warming, with similar sensitivities to changes in $\left[\mathrm{CO}_{2}\right]$ (Fig. S7).

\section{Crop and climate model uncertainty}

286 The range of crop model responses under SSP585 (mean across climate models) is

287 substantially larger than the range introduced by the five climate models (mean across crop

288 models; Fig. 1). However, for all crops and RCPs, the uncertainty associated with the five

289 CMIP6 climate models has increased compared to the five climate models sampled in GC5. In

290 turn, the fraction of total variance induced by the crop models is substantially reduced for all

291 crops in GC6 (for maize from 97 to 69\%; Fig. 8), which highlights that the crop response

292 became more consistent, even though the number of crop models increased. Absolute variance

293 induced by the climate models has increased for all crops (Fig. 8),

294 which is explained by a wider distribution of climate sensitivities tracked by the five CMIP6

295 GCMs (Table S1, S2), but also by higher [CO $\mathrm{CO}_{2}$ assumed in CMIP6 (Fig. 2). In this sample,

296 UKESM1 is the most pessimistic GCM for both RCPs and all crops, the global mean warming

297 level by 2099 is about $2.6^{\circ} \mathrm{C}$ higher than in GFDL-ESM4, and the Transient Climate Response

298 is $1.2^{\circ} \mathrm{C}$ higher (see Table S1 for more details) ${ }^{6}$. Generally, the least pessimistic crop impacts

299 are found with MRI-ESM2 (Fig. 1).

300

301 Higher emission scenarios inflate the crop model uncertainty (SSP585), while the overall

302 climate- and crop model-induced uncertainty range in GC6 is of comparable size under SSP126 
303 (Fig. 1). Uncertainty in the $\mathrm{CO}_{2}$ effect causes much of the crop model uncertainty for wheat, 304 soybean, and rice (Fig. S8), yet the range of maize responses is not fundamentally reduced 305 without the $\mathrm{CO}_{2}$ effect. In line with physiological knowledge ${ }^{30}$, crop models mostly show the 306 smallest $\mathrm{CO}_{2}$ effects for $\mathrm{C}_{4}$ crops (maize) and much larger responses for $\mathrm{C}_{3}$ crops (wheat, 307 soybean, rice). However, the $\mathrm{CO}_{2}$ effects differ widely across crop models; the ensemble 308 median rainfed response is $19 \%$ for maize, $33 \%$ for wheat, $48 \%$ for soybean, and $37 \%$ for rice 309 by the year 2099 (Fig. S8), which is generally in line with field experiments given that model 310 simulations include nutrient limitations ${ }^{25,30}$. CYGMA and CROVER exhibit a strong peak-and311 decline $\mathrm{CO}_{2}$ response for some crops, resulting in negative $\mathrm{CO}_{2}$ effects for maize in CYGMA 312 after 2090 (Fig. S8). This is driven by increased water use efficiencies under elevated [ $\left.\mathrm{CO}_{2}\right]$, 313 eventually leading to adverse excess moisture effects in humid regions - a new feedback 314 represented primarily in CYGMA and underexplored in previous studies ${ }^{39}$.

316 In addition to the $\mathrm{CO}_{2}$ effect, climate change affects simulations of crop growth and 317 development in various ways. These include for example changed precipitation patterns, 318 extreme heat and drought events, and importantly, accelerated maturity. Higher temperatures 319 lead to faster phenological development and substantial reductions in the growing season 320 length in all crop models (Fig. S9), which in turn lead to complex processes affecting yield, 321 including shorter grain filling periods, smaller canopy, and reduction in photosynthesis. This 322 effect varies across models and additional work is needed to further narrow the range of crop 323 model responses ${ }^{40}$. After all, the standard deviation of simulated yield variability matches 324 observational data to a much higher degree in GC6 $(R=79 \%)$ than in GC5 $(R=44 \%)$, adding 325 to more realistic yield responses (Fig. S10). 
327 We introduce the concept of climate impact emergence to the field of agriculture impacts,

328 highlighting that major shifts in global crop productivity due to climate change are projected to 329 occur within the next twenty years, several decades sooner than estimates based on previous

330 model projections. The impact on crop productivity under SSP126 and SSP585 is largely similar

331 for the coming decade, which leaves little room for climate mitigation efforts. In light of the much

332 larger climate and crop model agreement for these short-term projections than for the late

333 century, the findings highlight challenges for food system adaptation faced with significantly

334 shorter lead times.

336 These CMIP6 multi-model crop yield projections suggest that climate change impacts on global 337 agriculture will be more pronounced than in GC5, with substantially larger losses for maize, 338 soybean, and rice and additional gains for wheat. This is supported by a generally more 339 consistent crop model ensemble. However, large uncertainties remain, particularly in TCIE 340 estimates - the standard deviation for global maize TCIE is 24 years (SSP585), which is 341 similar to estimates of temperature emergence ${ }^{12}$. Yet the signal is robust: More than $80 \%$ of the 342 GCM-GGCM combinations indicate TCIE for maize and wheat by late century across major 343 breadbaskets (SSP585). TCIE estimates based on different metrics qualitatively agree (e.g., 344 multi-model ensemble mean TCIE for maize is found in the year 2032, the median of individual 345 GCM x GGCM estimates in the year 2027, and the mean in the year 2036). Leaving one crop 346 model out at a time introduces a TCIE standard deviation of only 1.5 years for both maize and 347 wheat (SSP585). That said, time of emergence estimates are sensitive to the underlying 348 definitions (e.g., noise, pre-industrial or recent climate, smoothing approach, threshold 349 selection) and can push the emergence date earlier or later in time ${ }^{12,13,15,41}$. Absolute TCIE 
estimates are therefore more challenging to interpret than relative comparisons among regions,

351 crops, and especially the two ensemble projections GC5 and GC6.

Wheat yield increases are projected to level off by midcentury and part of the climate-crop

354 model ensemble indicates net losses under SSP585 by 2099 (Fig. 1, S1). Maize yield on the

355 other hand is projected to decline steadily, supported by higher model agreement than for

356 wheat. These general response differences are also in line with previous findings ${ }^{42}$. The more

357 pronounced response of the new projections can be explained primarily by higher equilibrium

358 climate sensitivities, higher $\left[\mathrm{CO}_{2}\right]$, and different crop model sensitivities per degree warming and

$359\left[\mathrm{CO}_{2}\right]$ changes. With regard to CMIP6, higher and wider-ranging climate sensitivities are

360 critically discussed and associated with differing parameterizations of cloud feedback and cloud-

361 aerosol interactions ${ }^{14,43-49}$. While better simulations of cloud liquid water contents and their

362 radiative behavior render the climate models more realistic, it is unclear whether these

363 improvements translate into more accurate estimates of equilibrium climate sensitivity (ECS)

364 and overall warming levels. Additional improvements of the GCMs, and the bias-adjustment and

365 downscaling methods used, result in better representations of extreme events and internal

366 variability ${ }^{10,47,50-52}$, which are critical for crop modeling. Higher $\left[\mathrm{CO}_{2}\right]$ in $\mathrm{CMIP6}$ are due to a

367 revised tradeoff between $\left[\mathrm{CO}_{2}\right]$ and $\left[\mathrm{CH}_{4}\right]$ resulting from updated observations and assumptions

368 in the MAGICC7.0 model ${ }^{53}$.

370 The GGCMI crop model ensemble has substantially changed and consists of revised and new

371 members. For example, LPJmL contributed to GC5 and has since been fundamentally improved

372 with the addition of the nitrogen cycle $^{54}$ and heat unit parameterization ${ }^{55}$. In addition, input data

373 and model harmonization have been improved, including growing season harmonization based

374 on a new crop calendar developed for this study (see Methods). A comprehensive attribution of

375 crop response differences between GC5 and GC6 to changes in climate forcing, crop model 
376 selection and sensitivities, and input data is not feasible. But standardized comparisons of

377 changes in cropland warming and $\left[\mathrm{CO}_{2}\right]$ indicate that for maize and wheat changes in crop

378 model ensemble sensitivities dominate the response, and for soybean and rice higher warming

379 levels and warming sensitivity explain much of the differences (Fig. 7, S7).

381 The new GCM bias adjustment, crop model advancement, improved input data, and a new crop

382 yield bias correction serve to substantially reduce the amount of variance induced by the crop

383 models compared to the climate models, rendering the new GC6 ensemble more balanced and

384 consistent than GC5 despite a larger ensemble size (12 crop models in GC6, 7 in GC5; Fig. 8).

385 In a similar vein, Müller et al. ${ }^{56}$ comprehensively compared crop yield uncertainties under all

386 CMIP5 and CMIP6 GCMs based on GGCMI crop model emulators ${ }^{57}$, confirming that CMIP6

387 introduces a wider range of yield responses with more pessimistic average impacts. In view of

388 improved model harmonization, inputs, and GGCM versions and performance, we consider

389 GC6 more reliable than GC5 - despite ongoing discussions on the temperature sensitivity in

390 CMIP6.

392 The wide range of $\mathrm{CO}_{2}$ effects across GGCMI models is generally in line with field

393 experiments ${ }^{25,58,59}$, but the broad range of simulated $\mathrm{CO}_{2}$ fertilization effects merits more

394 rigorous model testing at the process level, which in turn requires better reference data,

395 especially at high $\left[\mathrm{CO}_{2}\right]$ levels. Moreover, elevated $\left[\mathrm{CO}_{2}\right]$ boosts crop yield, but it may also affect

396 the nutritional content of the crops $^{60-62}$. Impacts related to excess moisture, water resource

397 limitations, and new distributions of pests and diseases may lead to additional regional biotic

398 stresses requiring follow-on analysis.

400 Cropping system adaptation can substantially reduce and even outweigh adverse climate 401 change impacts, for example by switching to other crops ${ }^{63}$ or better-adapted varieties ${ }^{27,64}$. 
402 Integrated into ISIMIP's wider cross-sector activities, GGCMI will systematically evaluate

403 farming system adaptation and changes in yield variability and extreme event impacts in

404 subsequent efforts.

405

406 In conclusion, the new generation of AgMIP's GGCMI provides the most comprehensive

407 ensemble of process-based future crop yield projections under climate change to date. The

408 degree to which even high mitigation climate change scenarios are projected to push global

409 farming outside of its historical regimes suggests that current food production systems will soon

410 face fundamentally changed risk profiles. Despite prevailing uncertainties, these ensemble

411 projections spotlight the need for targeted food system adaptation and risk management across

412 the main producer regions in the coming decades.

413 


\section{Figures}

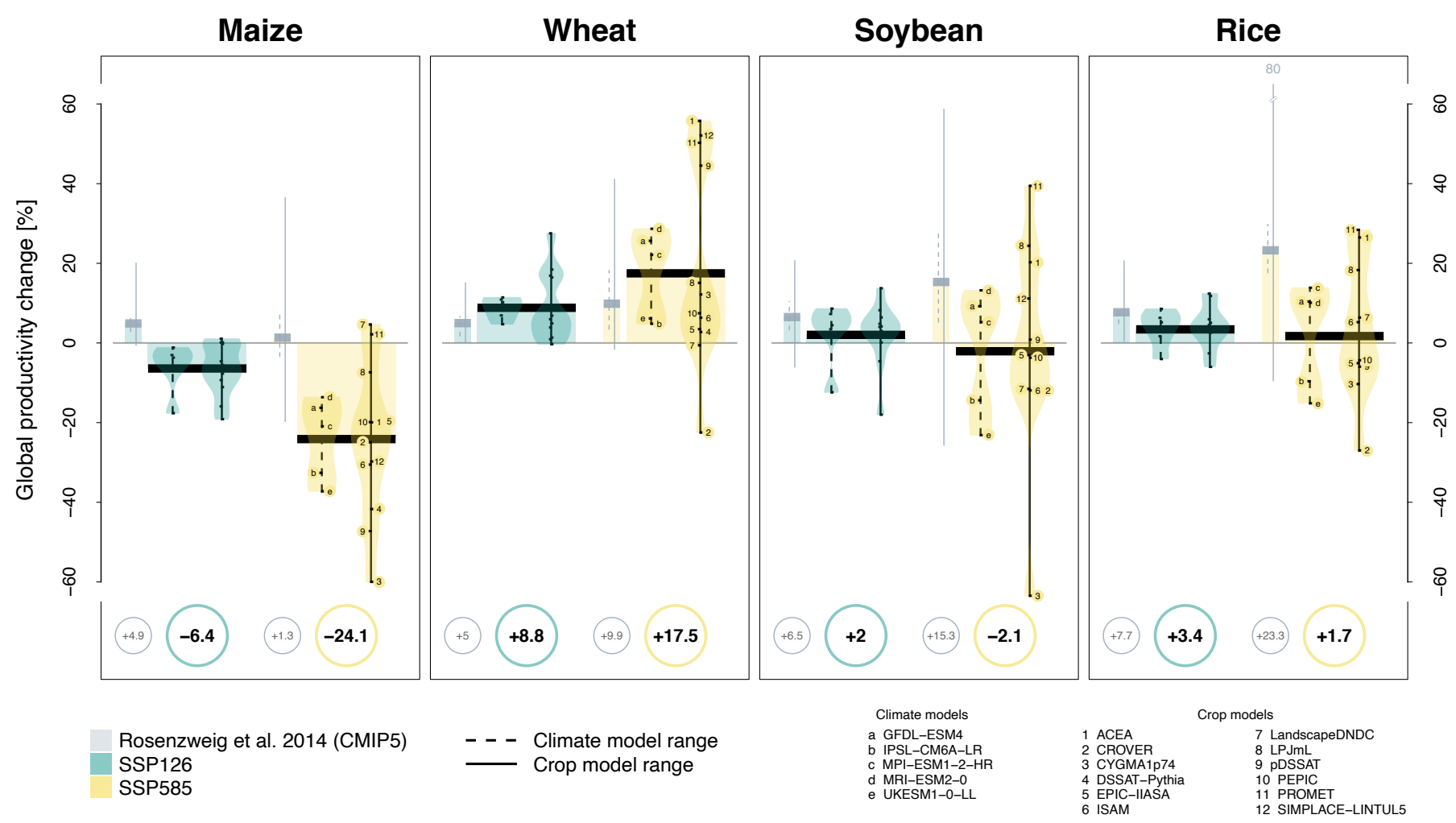

416 Fig. 1: Ensemble end-of-century crop productivity response. Global productivity changes (2069-2099 compared to 1983-2013) for SSP126 and SSP585 are shown as the mean across climate and crop models for the four major crops (highlighted by bullets underneath the plot). Whiskers indicate the range of individual climate model realizations (dashed line, as the mean across crop models), and the range across crop models (solid line, as the mean across climate models). Individual model results are indicated by the bullets along the whisker lines (for SSP585 only); violin shades additionally highlight the model distribution. For context, gray bars and whiskers reference previous GGCMI simulations based on CMIP5 (GC5; Rosenzweig et al. 2014) ${ }^{7}$ in the same way, without specifying individual models. Data are shown for the default $\left[\mathrm{CO}_{2}\right]$. Not all crop models simulate all crops, see Table S3 for details. 

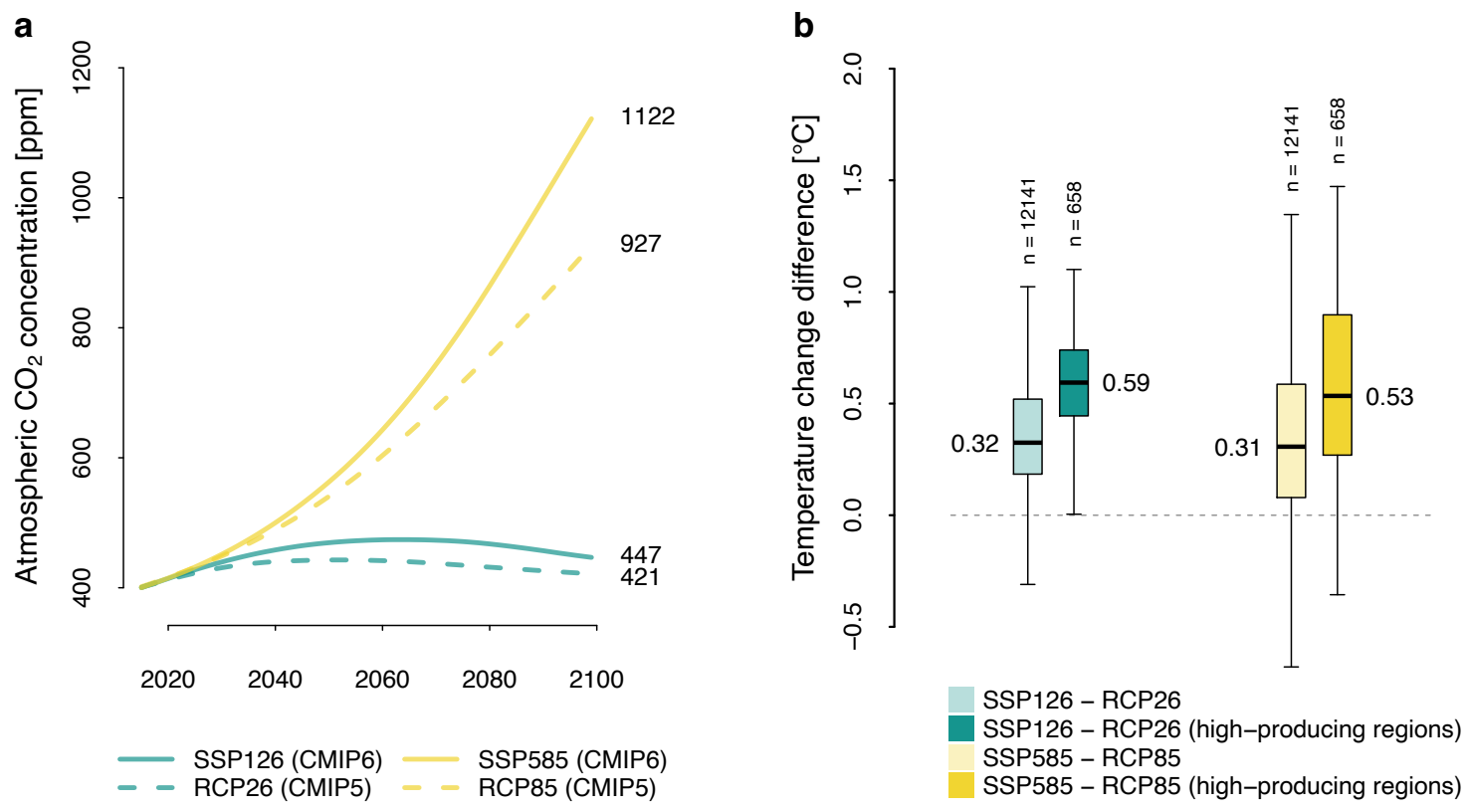

427 Fig. 2: Comparison of $\left[\mathrm{CO}_{2}\right]$ and temperature changes between CMIP5 and CMIP6. [CO $]$ pathways for 428 RCP26 and RCP85 in CMIP5 compared to SSP126 and SSP585 in CMIP6 (a). Box-and-whisker plots (b) 429 show the difference of the average maize growing season temperature changes [ $\left.{ }^{\circ} \mathrm{C}\right](2069-2099$ compared 430 to 1983-2013) between the CMIP6 and CMIP5 ensemble. Each ensemble is represented by the mean of 5 431 GCMs (Table S1 and S2) in each grid cell. CMIP6 and CMIP5 differences are separated for SSP126 (green) 432 433 and SSP585 (yellow) for all grid cells (maize production > 0; lighter shade) and for the highest-producing grid cells that together account for $50 \%$ of global production (darker shade). 

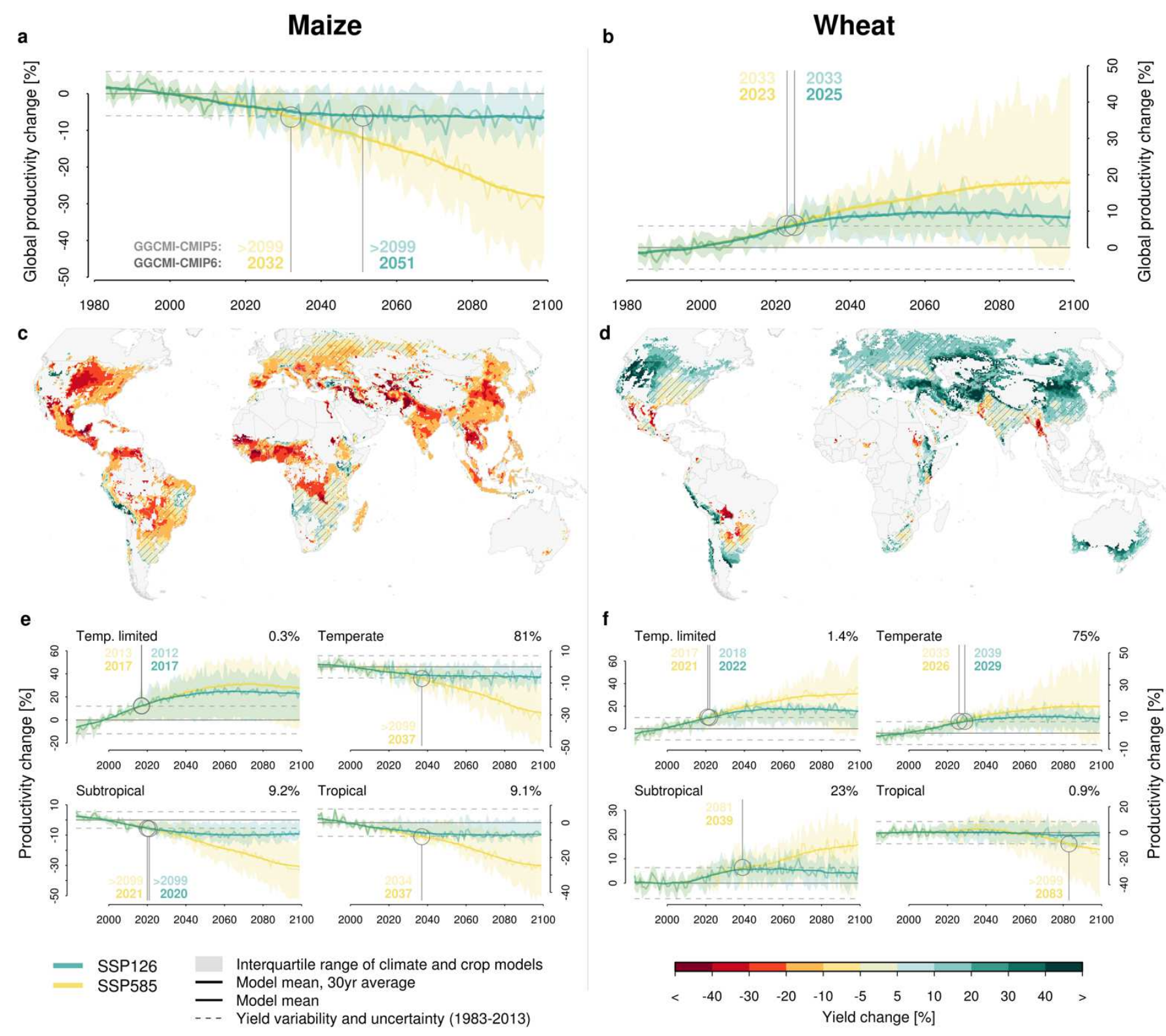

Fig. 3: Projections of global crop productivity for the $21^{\text {st }}$ century. For maize (a) and wheat (b), 436 productivity time series are shown as relative changes to the 1983-2013 reference period under SSP126 (green) and SSP585 (yellow). Shaded ranges illustrate the interquartile range of all climate-crop model 438 combinations (5 GCMs x 12 GGCMs). The solid line shows the median response (and a 25yr moving 439 average). Horizontal dashed lines mark the standard deviation of historical yield variability and model uncertainty (i.e., 'noise' from individual climate-crop model combinations) and open circles highlight the 'Time of Climate Impact Emergence' (TCIE), the year in which the smoothed climate change response emerges from the noise. For context, the TCIE calculated from $\mathrm{GC} 5{ }^{7}$ simulations is indicated in lighter shades above the TCIE based on GC6 (>2099 if no TCIE occurs by 2099). The maps (c, d) show median yield changes (2069-2099) under SSP585 across climate and crop models for current growing regions (>10 ha). Hatching indicates areas where less than $70 \%$ of the climate-crop model combinations agree on the sign of impact. Regional productivity time series $(\mathbf{e}, \mathbf{f})$ are similar to (a), but stratified for the four major Koeppen-Geiger climate zones (temperature limited, temperate/humid, subtropical, and tropical). The percentage of the total global production contributed by each zone is indicated in the top right corner of the insets. All data are shown for the default $\left[\mathrm{CO}_{2}\right]$ (see Fig. S4 for all four crops). 


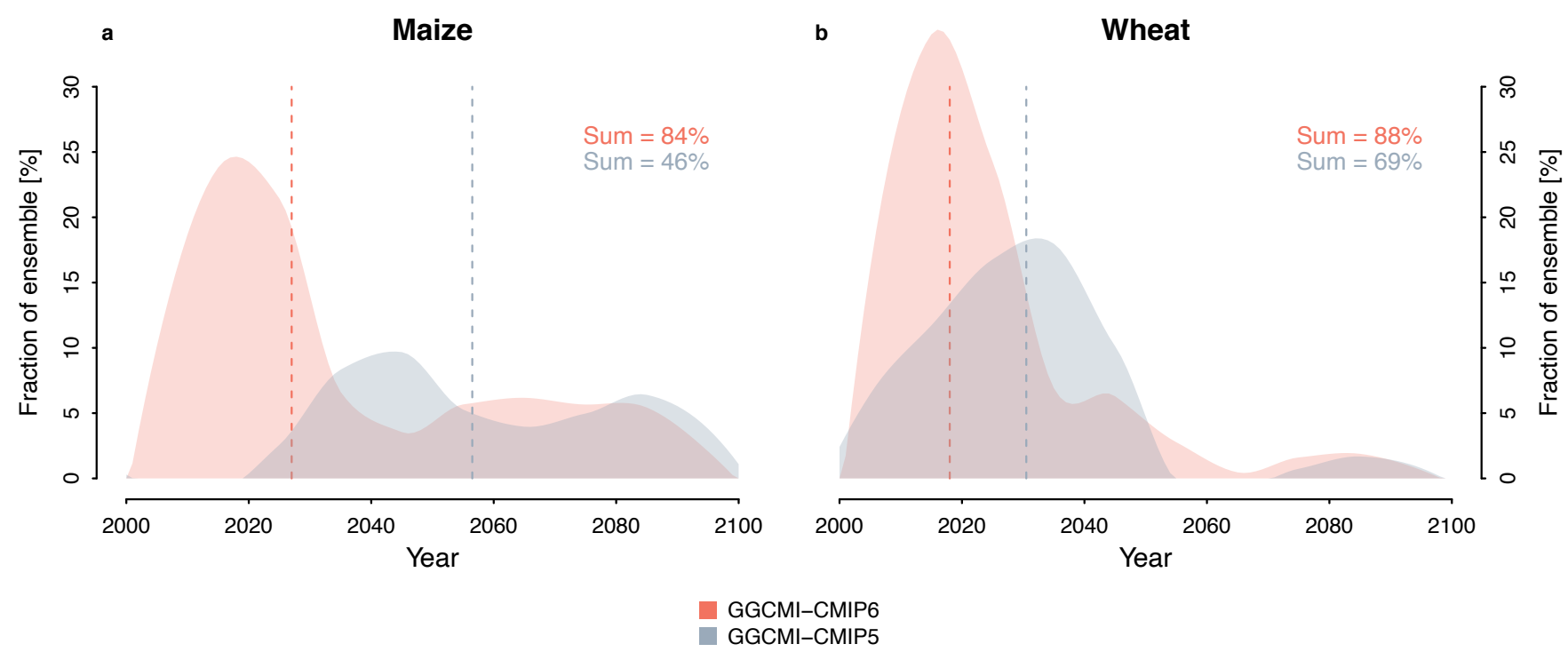

451 Fig. 4: Shift towards earlier and more pronounced climate impact emergence. Density plots of 452 individual TCIE estimates across the GCM x GGCM ensemble under SSP585 are shown for global maize 453 productivity (a; negative TCIE) and wheat (b; positive TCIE). Histogram counts are smoothed with a loess fit 454 (span=0.5) and shown as the fraction of the respective ensemble size. The GGCMI-CMIP6 ensemble 455 includes 12 crop models, GGCMI-CMIP5 includes 7 crop models; both comprise 5 GCMs. The total 456 ensemble fraction that shows TCIE by 2099 is indicated in the top-right corner ('Sum'). The ensemble 457 median TCIE is highlighted with vertical dashed lines. 

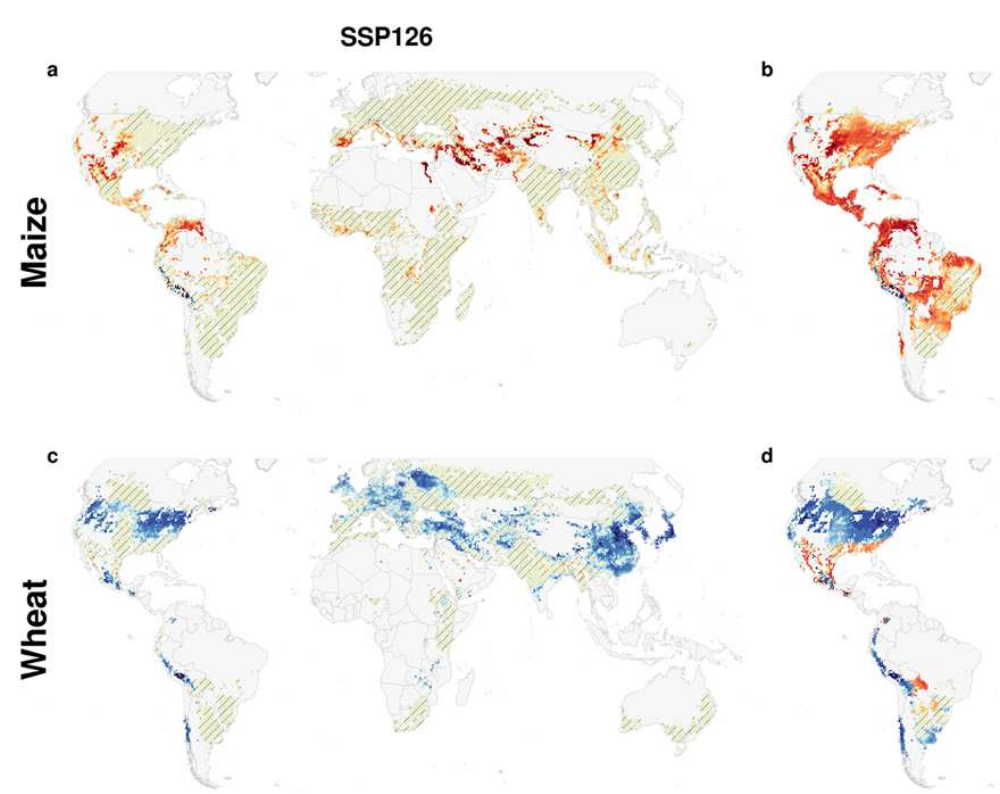

SSP585

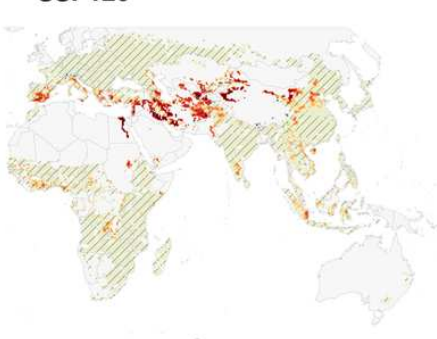

d

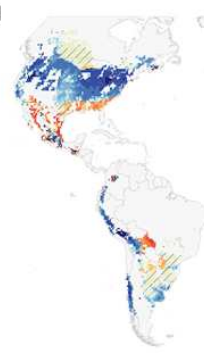

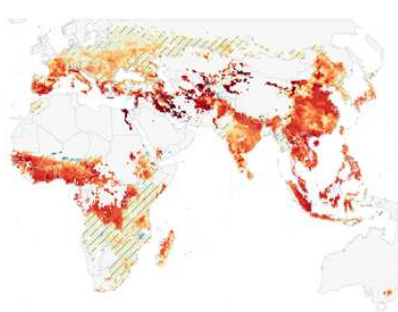
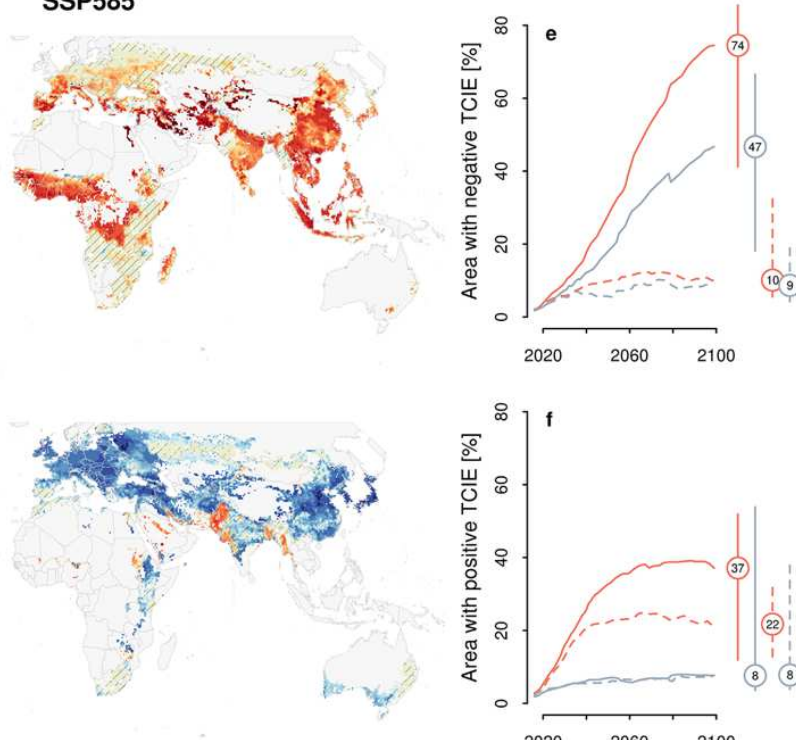
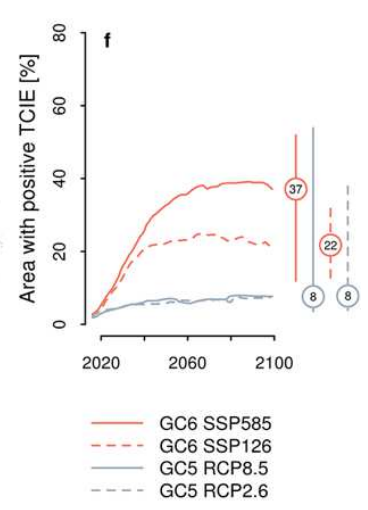

458

459

460

461

462

463

464

465

466

467

468

469

470

Fig. 5: Geographic patterns in TCIE. The maps show TCIE estimates for maize (a, b) and wheat (c, d) under SSP126 and SSP585 - calculated as the median of individual TCIE estimates from each climatecrop model combination. Hatching indicates areas in which less than $70 \%$ of the crop models agree on the emergence signal by 2099. See Figure S2 for the associated standard deviation of TCIE estimates, and Figure S3 for the signal-to-noise ratio. Panel (e) and (f) illustrate the annual percentage of the respective global cropland area affected by negative (maize) and positive (wheat) TCIE under SSP126 and SSP585, separated for results from $\mathrm{GC}^{7}$ and GC6. Vertical bars indicate the inter-quartile range of all climate-crop model combinations, with the median value in the circle. The maps show the first TCIE occurrence, even if the signal is reversed by late century (e.g., parts of India for wheat; compare with Fig. S3); estimates of the affected areas $(\mathbf{e}, \mathbf{f})$ account for signal changes. 


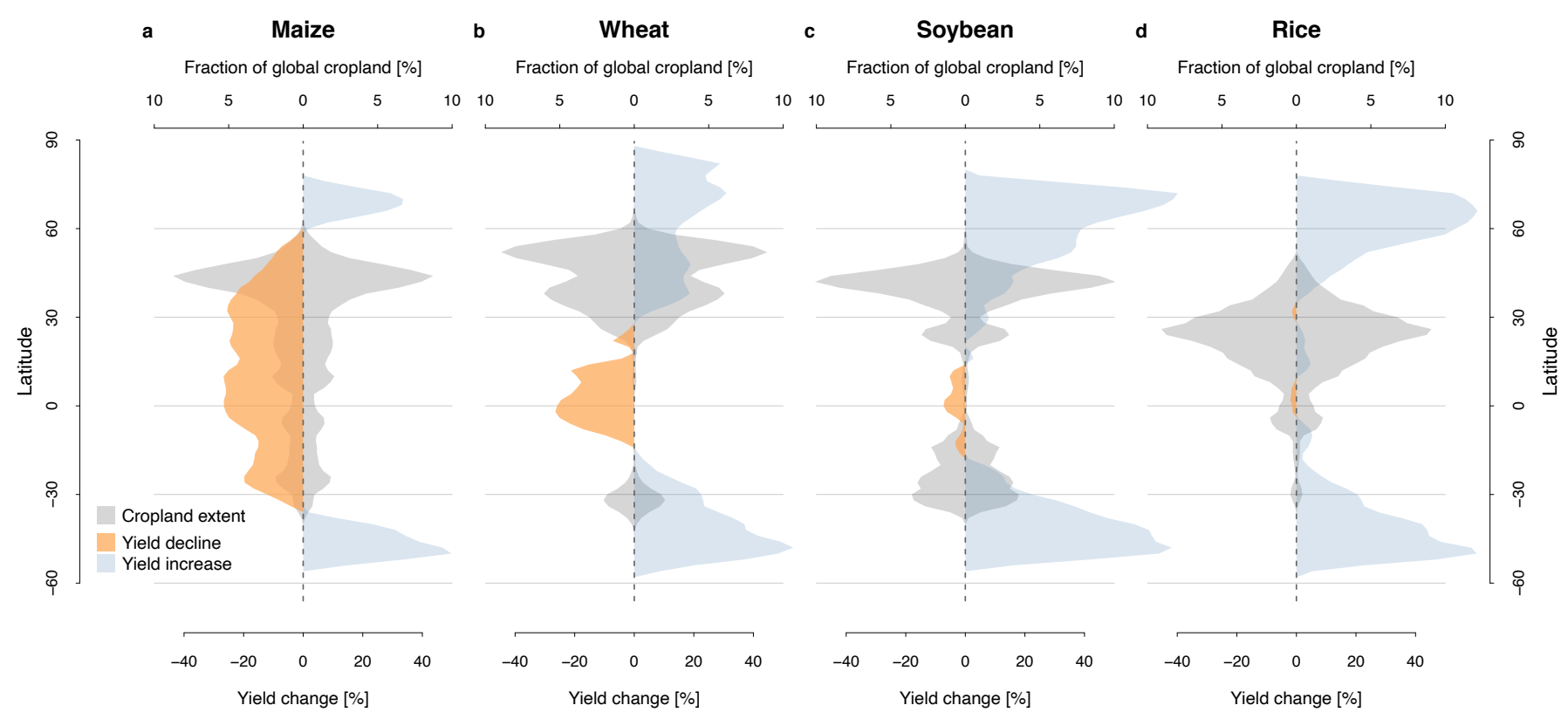

473 Fig. 6: Latitudinal profile of crop yield changes. Yield changes (SSP585, 2069-2099) are shown as 474 latitude averages for maize (a), wheat (b), soybean (c), and rice (d), based on crop simulations in all grid 475 cells, unconstrained by current cropland extent (bottom x-axis). For context, the current cropland extent is 476 shown across latitude bands as fractions of the crop-specific global extent (top x-axis; mirrored to allow overlaps with both positive and negative yield changes). Yield data are shown as the climate and crop model 478 median (marginal areas with yield lower than the $20^{\text {th }}$ percentile per crop are excluded). 


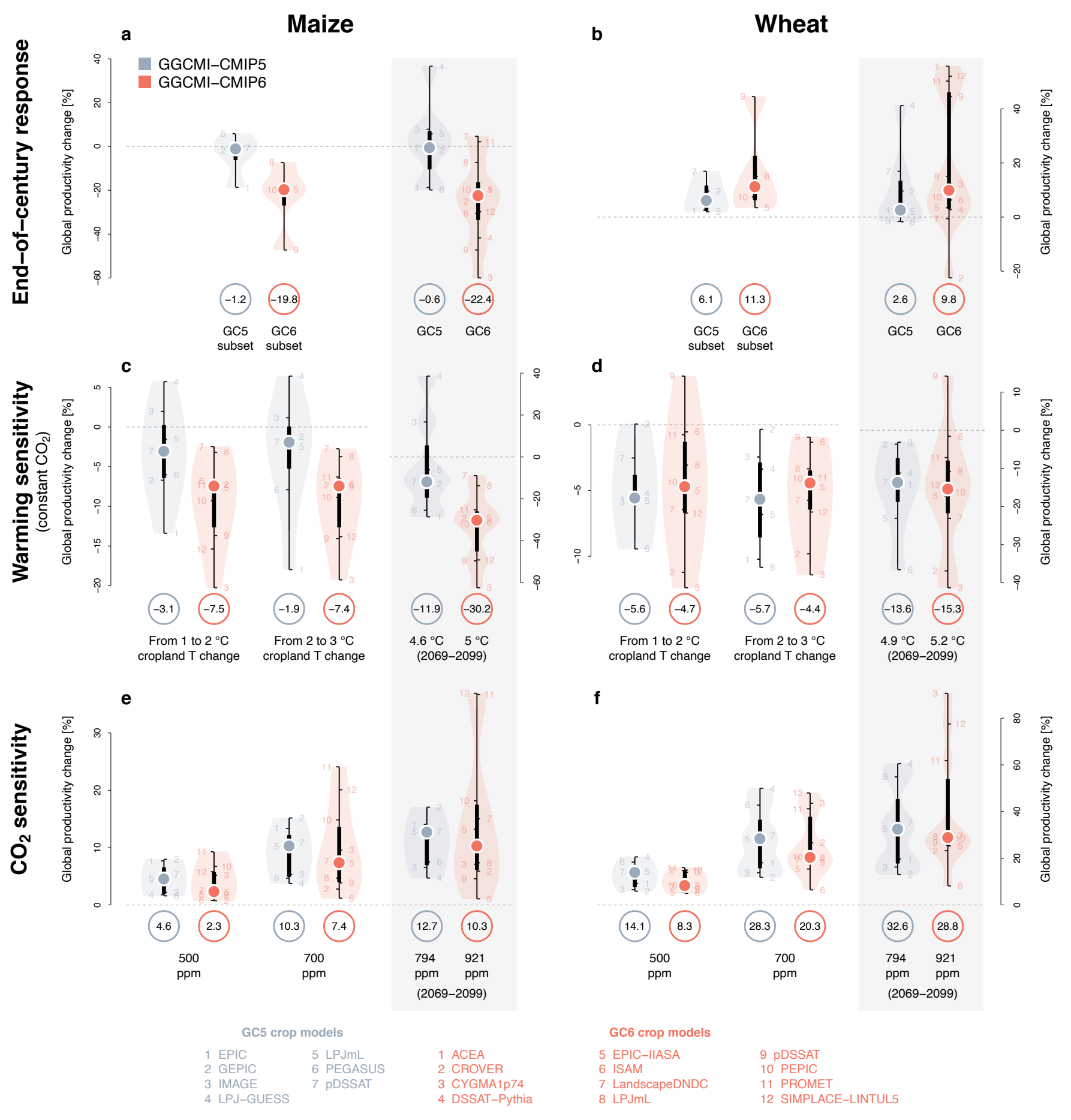

Fig. 7: Driver attribution of crop model responses. Projected end-of-century global productivity changes for maize (a) and wheat (b) under RCP8.5 (climate model mean) are shown for all members of the crop model ensemble GGCMI-CMIP5 (GC5) and GGCMI-CMIP6 (GC6), and for a subset of crop models that participated in both rounds (note substantial differences between model versions). The sensitivity to global mean warming (c, d) of the full ensembles is shown for temperature changes (over respective cropland areas per crop) from 1 to $2^{\circ} \mathrm{C}$, from 2 to $3^{\circ} \mathrm{C}$, and for the total change between 1983-2013 and 2069-2099. The warming sensitivity is based on $\left[\mathrm{CO}_{2}\right]$ held constant at the 2015 level but includes changes in other climate variables. The $\mathrm{CO}_{2}$ sensitivity $(\mathbf{e}, \mathbf{f})$ in $\mathrm{GC} 5$ and $\mathrm{GC} 6$ is shown at specific $\left[\mathrm{CO}_{2}\right]$ concentrations and for the 2069-2099 mean concentrations. Warming and $\mathrm{CO}_{2}$ sensitivities are calculated based on crop model responses over a 21-year window centered on the year in which a certain temperature change or $\left[\mathrm{CO}_{2}\right]$ concentration occurs in each climate model. Filled circles indicate the median crop model response, 
additionally highlighted by circled numbers underneath each plot. Black bars show the inter-quartile range and individual models are indicated by numbers. Note that both panel $\mathbf{c}$ and $\mathbf{d}$ include two different legends. See Figure $\mathrm{S} 7$ for soybean and rice results. ACEA and DSSAT-Pythia have not submitted simulations for the constant $\left[\mathrm{CO}_{2}\right]$ setting and are excluded from panel c-f.

495

496

497

498

499

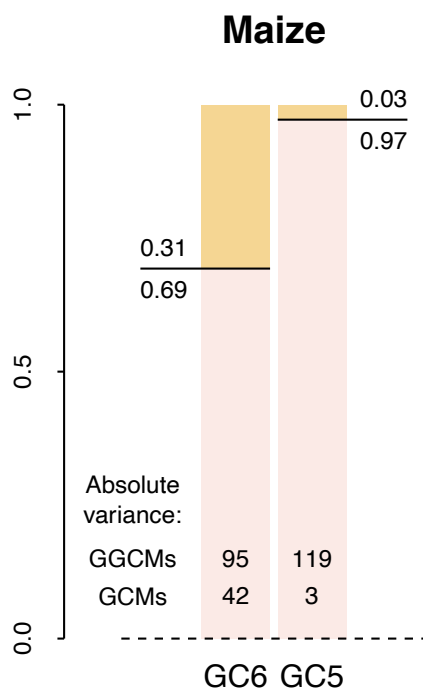

GC6 GC5
Wheat
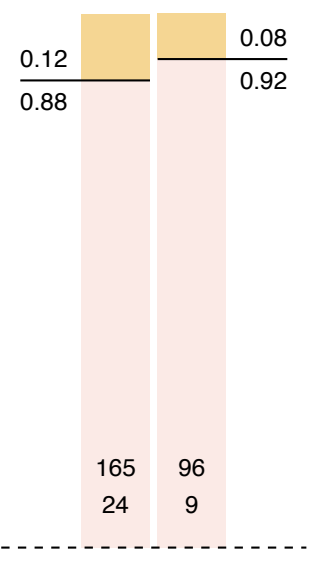

GC6 GC5
Soybean

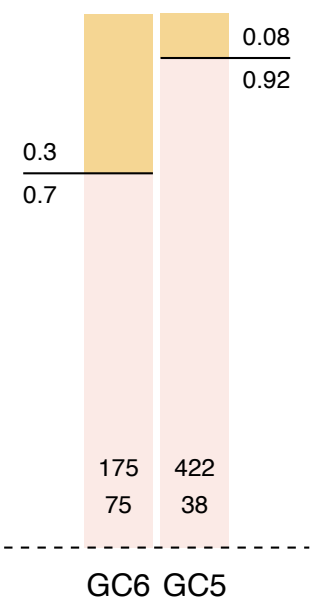

GC6 GC5
Rice

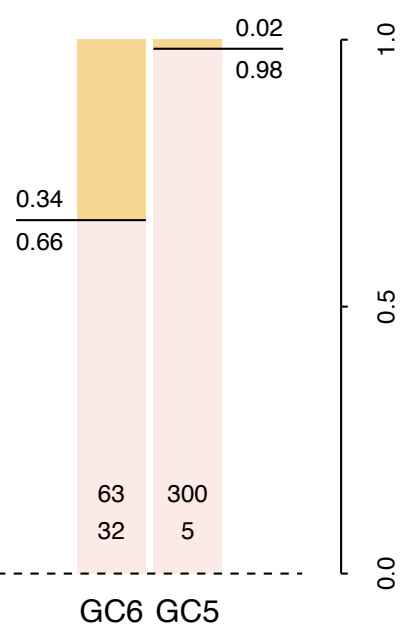

Variance induced by GCMs

Variance induced by GGCMs

Fig. 8: Variance decomposition of ensemble projections. Stacks show the fraction of total variance of mid-century crop production changes (2030-2070 mean) induced by the climate model ensemble (GCMs; yellow) and by the crop model ensemble (GGCMs; pink), for GGCMI-CMIP6 (GC6) and GGCMI-CMIP5 (GC5), respectively. Variance fractions are normalized by the variance cross term to be additive. The absolute variance introduced by GGCMs and GCMs is indicated at the base of each stack. The GCM ensemble has 5 members in both cases, the GGCM ensemble has 12 members in GC6 and 7 members in GC5, which further highlights that the crop model response became more consistent in GC6 compared to 508 the climate model uncertainty. 


\section{Methods}

\section{Time of emergence metric}

511 We define Time of Climate Impact Emergence (TCIE) as the year in which the smoothed climate

512 change signal ('signal') exceeds the underlying internal variability and model uncertainty ('noise'). The

513 signal is the multi-model ensemble mean crop productivity change against the 1983-2013 reference

514 period (smoothed with a 25-yr moving window). Noise is defined as the standard deviation of simulated

515 historical variability of crop productivity across all individual GCM x GGCM combinations (1983-2013).

516 TCIE is the first year in which the signal emerges from the noise, i.e., when the signal-to-noise ratio

517 becomes greater than 1. Similar time of emergence definitions have been used in previous

518 studies ${ }^{\text {e.g.10,13,68,69. }}$. Historical productivity time series are not detrended as we hold all management

519 factors constant throughout the simulations. To assess TCIE uncertainties, we calculate TCIE also for

520 each individual climate-crop model realization as suggested by Hawkins and Sutton $2012^{12}$, and we

521 analyze the distribution of the individual estimates (including mean, median, inter-quartile range, and

522 SD). We find that the multi-model ensemble mean TCIE usually occurs between the median and the

523 mean of individual TCIE estimates. For example, global-level maize production under RCP8.5 shows a

524 multi-model ensemble mean TCIE in year 2032, the median of individual estimates occurs in year 2027,

525 the mean in year 2036. Wheat shows the same pattern and results are qualitatively the same across

526 the different methods. To test the robustness of results in another way, we calculate the multi-model

527 ensemble mean TCIE iteratively while removing one crop model at a time. The SD of this distribution at

528 global level is marginal; 1.5 years for both maize and wheat under RCP8.5. As a final metric, we also

529 compare the number of climate and crop model combinations that show an emergence signal by the

530 end of the century. We calculate TCIE at global level, for different Koeppen-Geiger climate zones, and

531 for individual grid cells. Earlier TCIE is generally found for larger spatial scales as the variance of

532 internal variability decreases with averaging. For additional discussions see for example references ${ }^{11-}$ $533 \quad 13$ 


\section{ISIMIP climate input datasets}

535 GGCMI simulation efforts for CMIP6 impact assessment are aligned with the ISIMIP ${ }^{3}$ activity in which

536 GGCMI represents the agriculture sector. Key modeling inputs such as information on climate, land

537 use, fertilizer input, soils, among others, are harmonized across various research sectors. CMIP6

538 climate model outputs are centrally bias-adjusted and downscaled by the ISIMIP framework to provide

539 climate-input datasets on a daily regular $0.5^{\circ} \times 0.5^{\circ}$ global grid. The bias-adjustment method employs a

540 quantile mapping approach and uses the observational W5E5 v1.0 dataset ${ }^{67,68}$. This historical dataset

541 compares favorably with climatic forcing datasets that have been used previously by AgMIP GGCMI ${ }^{69}$.

542 The new quantile-mapping method adjusts biases and preserves trends in all quantiles of the

543 distribution of simulated daily climate model outputs; for more details see Lange $(2019)^{10}$. To lower the

544 barrier for participation in this study we provide climate input data for five CMIP6 GCMs: GFDL-ESM4,

545 IPSL-CM6A-LR, MPI-ESM1-2-HR, MRI-ESM2-0, UKESM1-0-LL (see Table S1 for further details). The

546 GCM selection is based on data availability at the time of selection, performance in the historical period,

547 structural independence, process representation and equilibrium climate sensitivity (ECS). The five

548 GCMs are structurally independent in terms of their ocean and atmosphere model components and

549 overall they represent the range of ECS across the full CMIP6 ensemble, including three models with

550 below-average ECS (GFDL-ESM4, MPI-ESM1-2-HR, MRI-ESM2-0) and two models with above-

551 average ECS (IPSL-CM6A-LR, UKESM1-0-LL) ${ }^{8}$. ECS and transient climate response (TCR) for all

552 GCMs used are listed in Table S1. The mean and standard deviation (SD) of both ECS $\left(\right.$ mean $=3.7^{\circ} \mathrm{C}$,

$553 \mathrm{SD}=1.1)$ and $\mathrm{TCR}\left(\right.$ mean $\left.=2.0^{\circ} \mathrm{C}, \mathrm{SD}=0.5\right)$ across the five $\mathrm{GCMs}$ used here precisely match the

554 mean and SD across the full CMIP6 ensemble with 38 members (Table S1 and S2), much better than

555 in GC5, although the range of ECS in the CMIP6 ISIMIP models is larger than in the CMIP5 ISIMIP

556 models.

557

558 The daily weather variables at a $0.5^{\circ}$ spatial resolution that are used as input for the crop models

559 include: daily mean, minimum, and maximum 2-m air temperature ( $T$, Tmin, and Tmax, respectively 
$560\left[{ }^{\circ} \mathrm{C}\right]$ ), daily total precipitation (P $\left.[\mathrm{mm}]\right)$, and daily mean shortwave and longwave radiation (SR and LR $561[\mathrm{~W} / \mathrm{m} 2])$.

562 GGCMI Phase 3 crop modeling protocol

563 Bias-adjusted climate model projections are used to drive transient crop model simulations, i.e.,

564 uninterrupted runs for the historical (1850-2014), and future (2015-2100) time period. Potential future

565 trajectories are represented by SSP1 with RCP2.6 (here SSP126) and SSP5 with RCP8.5 (here

566 SSP585). Therefore, each crop model performs 20 future simulation runs for each crop (5 GCM $\times 2$

567 RCP $\times 2\left[\mathrm{CO}_{2}\right]$ settings). Note that in this study any socio-economic forcing or adaptation effort

568 associated with the SSP storylines is held constant at the year 2015 level to isolate the climate signal

569 (i.e., year 2015 land-use, fertilizer application, growing seasons, crop cultivars, but also $\mathrm{NO}_{3}$ and $\mathrm{NH}_{4}$

570 deposition rates, are used in years after 2015). To help isolate yield effects associated with the $\mathrm{CO}_{2}$

571 fertilization effect, all crop model simulations are run for two separate assumptions: i) transient $\left[\mathrm{CO}_{2}\right]$ in

572 line with the respective RCP ('default [CO $]$ '), and ii) $\left[\mathrm{CO}_{2}\right]$ concentration held constant at the 2015 level

573 at 399.95 ppmv ('constant $\left[\mathrm{CO}_{2}\right]$ '). Differences between the two $\left[\mathrm{CO}_{2}\right]$ levels are not a measure of $\left[\mathrm{CO}_{2}\right]$

574 uncertainty, as there is no plausible climate change scenario without increasing $\left[\mathrm{CO}_{2}\right]^{22}$. Instead, this

575 setup is used to quantify the size of the $\mathrm{CO}_{2}$ fertilization effect. All simulations are carried out at the $0.5^{\circ}$

576 global grid. In addition to the GCM forcing, we include historical simulations based on the reanalysis

577 product GSWP3-W5E5 v1.067,68 for each crop model and crop to better evaluate crop model

578 performance against observational data.

579

580 We focus on the four major global grain crops, that is, maize (Zea mays L.), wheat (Triticum sp. L.), rice

581 (Oryza sativa L.), and soybean (Glycine max L. Merr.). Wheat is simulated as winter and spring wheat

582 individually; grain and silage maize are not distinguished. These four main crops contribute $90 \%$ of

583 today's global caloric production of all cereals and soybean ${ }^{70}$. 
585 All crops are simulated under both rainfed conditions and full irrigation (where soil moisture is set to

586 field capacity every day, without constraints to water availability) in all grid cells — independent of the

587 current cropland distribution. The physical cropland extent is applied in post-processing based on the

588 MIRCA2000 (Monthly Irrigated and Rainfed Crop Areas around the year 2000) reference dataset ${ }^{71}$ and

589 irrigated fractions are adapted from Siebert et al. (2015) ${ }^{72}$; both are held constant over time.

590

591 Soil moisture and soil temperature for various soil layers are calculated by most crop models in a

592 transient way, that is, without reinitializing at the beginning of each year. All models use a classic

593 phenological heat sum approach to determine physiological stages between planting and maturity. Heat

594 unit accumulation can be modified by the sensitivity to day length (photoperiod) and for winter wheat it

595 is stalled until vernalization requirements are reached, that is, the exposure to cold temperatures before

596 anthesis. Planting dates (see section 'Crop calendar and crop varieties' below) are constant over time

597 but the heat sum approach leads to different growing season lengths depending on the daily

598 temperature distribution in each growing season. Except for rice, we simulate only one growing season

599 per calendar year. The first and last years of the transient runs are removed from crop model

600 simulations due to partially incomplete growing seasons. Simulations in grid cells with a growing

601 season length less than 50 days are removed, as are simulations resulting in premature harvest (i.e.,

602 accumulated heat units $<80 \%$ of required heat units and applies only to those models that can provide

603 such outputs).

604

605 The harmonization of crop models includes the required use of a central crop calendar product (new

606 development for this study, see below), fertilizer inputs, and soil information. Additional protocol

607 characteristics are recommended but not required, as not all models can address all features (see

608 below).

609

610 Simulation protocols determine mineral and organic fertilizer [kg N/ha] inputs per crop and grid cell.

611 Mineral fertilizer (ammonium nitrate; $\mathrm{NH}_{4} \mathrm{NO}_{3}$ ) application is crop-specific and is derived from the $\mathrm{LUH} 2$ 
612 product $^{73,74}$, harmonized by ISIMIP. Manure application inputs (C:N ratio of 14.5) are grid cell specific,

613 but constant across crops $^{75}$. All other nutrients are considered non-limiting. Fertilizer scheduling follows

614 a simple assumption with $20 \%$ applied at sowing and $80 \%$ applied when $25 \%$ of the heat units required

615 to reach maturity are accumulated. As all other management aspects, fertilizer application is held

616 constant throughout the simulation period. Atmospheric $\mathrm{N}$ deposition is considered, separating $\mathrm{NH}_{\mathrm{x}}$ and

$617 \mathrm{NO}_{\mathrm{y}}$, based on Tian et al. $(2018)^{76}$ and held constant at the year 2015 level.

618

619 Soil input is harmonized across crop models for the first time in GGCMI, derived from the Harmonized

620 World Soil Database (HWSD) ${ }^{77}$. While the same HWSD dataset is used across ISIMIP sectors, in this

621 study we employ a different algorithm to aggregate the data to $0.5^{\circ}$ in order to be cropland specific. The

622 pDSSAT model uses the Global Soil Data set for Earth system modeling (GSDE) $)^{78}$ and DSSAT-Pythia

623 uses the Global High-Resolution Soil Profile Database for Crop Modeling Applications ${ }^{79}$ due to

624 difficulties in retrieving all soil parameters from HWSD.

625

626 Finally, the following management aspects are encouraged to be harmonized across crop models, but

627 are not accounted for by all teams: tillage (2 tillage events, planting day and harvest day, $200 \mathrm{~mm}$

628 depth, full inversion), residues (70\% of above-ground residues removed), no pest and disease damage,

629 no soil erosion, and no cover crops. Except for rice and wheat, which are simulated for two separate

630 growing seasons, we do not consider multi-cropping systems or crop rotations. Inputs are provided for

63118 different crops globally, but most crop models can only simulate the major crops, which we focus on

632 in this study. All socio-economic and farm management input data are publicly available via

633 www.isimip.org.

634 Participating GGCMI crop models

635 Twelve process-based global crop models participate in this study: ACEA, CROVER, CYGMA1p74,

636 DSSAT-Pythia, EPIC-IIASA, ISAM, LandscapeDNDC, LPJmL, pDSSAT, PEPIC, PROMET,

637 SIMPLACE-LINTUL5 (see Table S3 for further details and references). The full ensemble, therefore, 
638 consists of roughly 240 future crop model simulations per crop plus one historical reference run for

639 each crop and climate model and one historical reanalysis run per crop model. Due to computational

640 constraints, ACEA has only run GCMs UKESM1-0-LL and MRI-ESM2-0 so far, and DSSAT-Pythia has

641 not yet run UKESM1-0-LL. ACEA and DSSAT-Pythia have not yet finished simulations for the constant

$642\left[\mathrm{CO}_{2}\right]$ setting.

643

644 All crop models are considered independent. LPJmL, pDSSAT, EPIC-IIASA, PROMET, and PEPIC

645 have participated in previous GGCMI protocols ${ }^{7,80-82}$, and while the other models are new GGCMI

646 ensemble members, they have been thoroughly evaluated individually (see references in Table S3). In

647 order to participate in this study, each model was required to go through a benchmark performance

648 evaluation for the historical period based on GSWP3-W5E5 reanalysis data (results available upon

649 request). An overview of the degree to which the GC6 crop models explain observed inter-annual yield

650 variability is presented in Figure S11. For the top five producer countries per crop, the ensemble mean

651 generally shows higher performance in terms of correlation and root-mean-square error than the bulk of

652 individual models. Generally, explained variability in individual models is satisfactory for most maize,

653 wheat, and soybean main-producer countries. The metrics are lower for rice which also links to the fact

654 that the weather signal in (largely irrigated) rice is smaller than in other crops, and the overall observed

655 inter-annual variability in these rice producer countries is smaller than for the other crops. Since

656 management decisions (planting dates, crop rotations and areas, fertilizer application, irrigation, etc.)

657 are held constant over time, the crop models can only capture the interannual weather signal in

658 reported yields, which in general is much smaller in the tropics compared to mid- to high-latitude

659 regions. Additional in-depth GGCMI model comparison and evaluation is presented by Müller et al.

$660(2017)^{81}$. Overall, crop model performance evaluation based on historical yield variability provides

661 limited insight into the models' capability to project future yield impacts ${ }^{83}$.

663 Since GCM-based crop model simulations are difficult to compare with observed inter-annual yield

664 levels (e.g., the 1988 drought does not necessarily occur in 1988 in the GCM), we compare the overall 
665 range of simulated and observed yield variability across the historical reference period. The standard 666 deviation of observed national yield variability is matched to a substantially higher degree in GC6 (R = $66779 \%$, RMSE $=0.11)$ than in GC5 $(\mathrm{R}=44 \%$, RMSE $=0.17)$, which is indicative of more realistic yield 668 responses in GC6 (Fig. S10). These improvements are linked to a combination of factors, including 669 different internal variability in CMIP6, new GCM bias-adjustment method, improved crop model 670 ensemble, new crop yield bias-correction, and improved crop model inputs. The match with observed

671 yield variability using GC6 simulations based on GSWP3-W5E5 reanalysis data is only slightly better (R $672=87 \%$, RMSE $=0.09)$ than with GCM-forced simulations, which highlights that the CMIP6 GCMs do not 673 introduce substantial errors in terms of historical variability (Fig. S10).

674

675 While the models generally reproduce yield declines in extreme years, adverse impacts of excess water 676 on crop growth due to lower aeration, waterlogging, and nitrogen leaching are generally

677 underrepresented in current global crop models ${ }^{39}$. As an exception, the crop model CYGMA accounts 678 for effects due to excess moisture stress ${ }^{84}$. ACEA, EPIC-based, and DSSAT-based crop models also 679 have processes related to waterlogging and root aeration but associated stresses occur rarely and 680 foremost on sensitive soils ${ }^{85}$. Many models do not handle direct effects of extreme heat (e.g., on leaf 681 senescence, pollen sterility; see Table S3) ${ }^{3}$. Individual model responses to elevated $\left[\mathrm{CO}_{2}\right]$ are shown in 682 Figure 7 and S8 and discussed in the main text. The ISAM model requires sub-daily weather data and 683 therefore uses CRU-National Centers for Environmental Prediction (CRUNCEP) diurnal factors to 684 convert daily bias-adjusted climate model data to diurnal data. The PROMET model also requires sub685 daily weather data and uses ERA5-derived diurnal factors to convert climate model data to diurnal 686 inputs; it also uses WFDE5 instead of GSWP3-W5E5 for reanalysis simulations.

688 All models use spin-up simulations of various lengths to reach soil and carbon pool equilibrium. EPIC689 IIASA uses dynamic soil handling during spin-up to generate soil attributes. Subsequently these are 690 used as an input in the actual simulations with static soil handling, i.e. annual re-initialization of all soil 691 attributes (including soil organic matter fractions and soil texture among others) except mineral nutrient 
692 pools, temperature, and soil moisture. The models do not account for human management intervention

693 other than fertilizer application, irrigation, seed selection, growing periods, and basic field management

694 such as tillage and residue removal.

695

696 All models follow a phenology calibration with respect to grid cell-specific cultivar parameterizations

697 (i.e., phenological heat units) based on the respective crop calendar and weather forcing (Table S3).

698 Yield calibration is not harmonized across crop models and each team follows their individual protocol,

699 including grid cell-specific calibration against SPAM ${ }^{86}$ reference yields (e.g., pDSSAT), various site-

700 specific efforts based on field experiments (e.g., ISAM), and calibrations with national FAO ${ }^{70}$ statistics

701 (e.g., PEPIC).

702 Crop yield bias correction

703 Crop production is calculated as yield times harvested area of the respective crop. We omit grid cells

704 with <10 ha cropland area for each crop. To compare results across crop models, but also to represent

705 realistic overall crop production estimates and spatial pattern, we calculate fractional yield changes

706 from each individual crop model simulation between the historical reference period (1983-2013) and the

707 respective future projection and multiply these with a spatially explicit $\left(0.5^{\circ}\right)$ observational yield

708 reference dataset (see Fig. S14 in ref. ${ }^{87}$ ). SPAM2005 (Spatial Production Allocation Model 2005) (s $^{88}$

709 used as the main reference yield data as it separates rainfed and irrigated systems. Grid cells with

710 missing SPAM2005 yield data but with >10 ha MIRCA2000 harvested area are gap-filled with Ray et al.

$711(2012)^{89}$ yield data; both SPAM2005 and Ray et al. represent the time period 2003 to 2007.

712 Winter and spring wheat separation

713 While winter and spring wheat are simulated separately by the crop models covering all land areas, our

714 analyses distinguish winter and spring wheat harvested areas using a rule-based approach. We

715 assume that winter wheat is grown in a specific grid cell if: i) the average temperature of the coldest

716 month is between $-10^{\circ} \mathrm{C}$ and $+7^{\circ} \mathrm{C}$, ii) the growing season length exceeds 150 days, and iii) the growing 
717 season includes December (Northern Hemisphere) or July (Southern Hemisphere). These assumptions

718 are slightly modified from the rule set in MIRCA2000 71 ; we use $7^{\circ} \mathrm{C}$ instead of $6^{\circ} \mathrm{C}$ as the upper

719 temperature threshold to allow for more winter wheat in Argentina, South Africa, and Australia, but also

720 to extend winter wheat in the US slightly towards the south (Fig. S12). This modification is done to

721 better represent the winter wheat mega environments used by $\mathrm{CIMMYT}^{90}$. The winter and spring wheat

722 rule set is also used to separate wheat crop calendars in case the two are not distinguished in the

723 original crop calendar data. In line with other cropland areas as well, winter and spring wheat areas are

724 held constant over time.

\section{Crop calendar and crop varieties}

726 We provide planting and maturity dates for each crop in each grid cell, separate for rainfed and irrigated

727 systems, based on a new observational crop calendar product. See section 'GGCMI crop calendar' and

728 Fig. S13-S15 in the Supplement for details. Growing season inputs are static over time throughout the

729 historical and future time period to avoid confounding trends. Each model calculated required reference

730 heat units to reach physiological maturity for each crop in each grid cell by averaging annual heat sums

731 over all growing seasons between 1979-2010.

732 Koeppen-Geiger climate class aggregation

733 Koeppen-Geiger climate zones ${ }^{91}$ are aggregated to $0.5^{\circ}$ spatial resolution and the 32 individual classes

734 are aggregated to the following four main climate types: temperature-limited

735 ("Dfc","Dfd","Dsc","Dsd","Dwc","Dwd","ET","EF","H","BSk"), temperate/humid

736 ("Csb","Cfa","Cfb","Cfc","Csc","Cwa","Cwb","Cwc","Dfa","Dfb","Dsa","Dsb","Dwa","Dwb"),

737 subtropical/Mediterranean ("Csa","BSh","Af","Am","As","Aw"), and tropical/other (all other classes).

\section{Map projection and smoothing}

739 Global maps are based on the Robinson projection ${ }^{92}$ and grid-level data are smoothed to improve

740 clarity and visual appearance. Smoothing is done by first resampling the raw data to 5 times finer 
resolution, followed by a $5 \times 5$ grid cell focal mean window aggregation. Map smoothing is done for

742 visualization purposes only and all analyses are based on the raw data.

743

744

745

746

747

748

749

750

751

752

753

754

755

756

757

758

759

760

761

762

763

764

765

766

767

768

769

770

771

772

773

774

775

776

777

778

\section{Acknowledgements}

J.J., A.C.R., C.R., and M.P.P. were supported by NASA GISS Climate Impacts Group and Indicators for the National Climate Assessment funding from the NASA Earth Sciences Division. J.J. received support from the Open Philanthropy Project and thanks the University of Chicago Research Computing Center for supercomputer allocations to run the pDSSAT model. Ludwig-Maximilians-Universität München thanks the Leibniz Supercomputing Center of the Bavarian Academy of Sciences and Humanities for providing capacity on the Cloud computing infrastructure to run the PROMET model. J.M.S. was supported by the German Federal Ministry of Education and Research (grant-number 031B0230A: BioNex-The Future of the Biomass Nexus). O.M. and J.F.S. were supported by funding from the European Research Council (ERC) under the European Union's Horizon 2020 research and innovation programme (Earth@lternatives project, grant agreement No 834716). J.A.F. and H.S. were supported by the NSF NRT program (grant no. DGE-1735359). J.A.F was supported by the NSF Graduate Research Fellowship Program (grant no. DGE-1746045). RDCEP is funded by NSF through the Decision Making Under Uncertainty program (grant \#SES-1463644). T.I. was partly supported by the Environment Research and Technology Development Fund (2-2005) of the Environmental Restoration and Conservation Agency and Grant-in-Aid for Scientific Research B (18H02317) of the Japan Society for the Promotion of Science. M.O. was supported by the Climate Change Adaptation Research Program of NIES, Japan. S.L. was supported by the German Federal Office for Agriculture and Food (BLE) in the framework of OptAKlim (grant no. 281B203316). S.R. acknowledges funding from the German Federal Ministry of Education and Research (BMBF) via the ISIpedia project.

\section{Author contributions}

J.J. and C.M. conceived the paper and coordinate GGCMI. J.J., C.M., and S.R. developed the simulation protocol. A.R. and C.R. coordinate AgMIP integration. C.M., J.J., J.B., O.C., B.F., C.F., K.F., G.H. T.I., A.J. N.K, T.L., W.L., S.M., M.O., O.M., C.P. S.R., J.S., J.S. R.S., A.S., T. S., F.Z. conducted crop model simulations, S.L. prepared climate data inputs, J.J. developed the manuscript and figures, all coauthors supported writing and discussion of the results.

\section{Data and materials availability}

All data needed to evaluate the conclusions in the paper are present in the paper and/or the Supplementary Materials. Model inputs are publicly available via https://www.isimip.org/ or from the corresponding author. Crop model simulations will be made public under the CCO license pending publication.

The authors declare no competing interest. This article contains supporting information online.

\section{References}

1. Mbow, C. et al. Food security. in Climate Change and Land: an IPCC special report on climate change, desertification, land degradation, sustainable land management, food security, and 
greenhouse gas fluxes in terrestrial ecosystems 1, 270 (2019).

2. Asseng, S. et al. Uncertainty in simulating wheat yields under climate change. Nat. Clim. Chang. 3, 827-832 (2013).

3. Wang, E. et al. The uncertainty of crop yield projections is reduced by improved temperature response functions. Nat. Plants 3, (2017).

4. Rosenzweig, C. et al. The Agricultural Model Intercomparison and Improvement Project (AgMIP): Protocols and pilot studies. Agric. For. Meteorol. 170, 166-182 (2013).

5. ISIMIP. The Inter-Sectoral Impact Model Intercomparison Project. 2021 Available at: https://www.isimip.org/.

6. Eyring, V. et al. Overview of the Coupled Model Intercomparison Project Phase 6 (CMIP6) experimental design and organization. Geosci. Model Dev. 9, 1937-1958 (2016).

7. Rosenzweig, C. et al. Assessing agricultural risks of climate change in the 21 st century in a global gridded crop model intercomparison. Proc. Natl. Acad. Sci. 111, 3268-3273 (2014).

8. Meehl, G. A. et al. Context for interpreting equilibrium climate sensitivity and transient climate response from the CMIP6 Earth system models. Sci. Adv. 6, 1-11 (2020).

9. O'Neill, B. C. et al. The Scenario Model Intercomparison Project (ScenarioMIP) for CMIP6. Geosci. Model Dev. 9, 3461-3482 (2016).

10. Lange, S. Trend-preserving bias adjustment and statistical downscaling with ISIMIP3BASD (v1.0). Geosci. Model Dev. 12, 3055-3070 (2019).

11. Hawkins, E. et al. Observed Emergence of the Climate Change Signal: From the Familiar to the Unknown. Geophys. Res. Lett. 47, (2020).

12. Hawkins, E. \& Sutton, R. Time of emergence of climate signals. Geophys. Res. Lett. 39, 1-6

801 (2012).

13. Kirtman, B. et al. Near-term climate change: Projections and predictability. Clim. Chang. 2013 Phys. Sci. Basis Work. Gr. I Contrib. to Fifth Assess. Rep. Intergov. Panel Clim. Chang. 9781107057, 953-1028 (2013).

14. Seneviratne, S. I. \& Hauser, M. Regional Climate Sensitivity of Climate Extremes in CMIP6 Versus CMIP5 Multimodel Ensembles. Earth's Futur. 8, 1-12 (2020).

15. Rojas, M., Lambert, F., Ramirez-Villegas, J. \& Challinor, A. J. Emergence of robust precipitation changes across crop production areas in the 21 st century. Proc. Natl. Acad. Sci. 116, 66736678 (2019). human tolerance. Sci. Adv. 6, (2020).

17. Park, C. E. et al. Keeping global warming within $1.5^{\circ} \mathrm{C}$ constrains emergence of aridification. Nat. Clim. Chang. (2018). doi:10.1038/s41558-017-0034-4

18. Liu, B. et al. Similar estimates of temperature impacts on global wheat yield by three 
independent methods. Nat. Clim. Chang. 6, 1130-1136 (2016).

816 19. Zhao, C. et al. Plausible rice yield losses under future climate warming. Nat. Plants 3, 1-5

817 (2016).

818 20. Zhao, C. et al. Temperature increase reduces global yields of major crops in four independent

819 estimates. Proc. Natl. Acad. Sci. 201701762 (2017). doi:10.1073/pnas.1701762114

820 21. Deryng, D. et al. Regional disparities in the beneficial effects of rising CO2 concentrations on 821 crop water productivity. Nat. Clim. Chang. (2016). doi:10.1038/nclimate2995

822 22. Ruane, A. C. et al. Biophysical and economic implications for agriculture of $+1.5^{\circ}$ and $+2.0^{\circ} \mathrm{C}$ 823 global warming using AgMIP Coordinated Global and Regional Assessments. Clim. Res. 76, 1782439 (2018).

825 23. Ahmed, M. et al. Novel multimodel ensemble approach to evaluate the sole effect of elevated 826 CO2 on winter wheat productivity. Sci. Rep. 9, 1-15 (2019).

827 24. Leakey, A. D. B., Bishop, K. A. \& Ainsworth, E. A. A multi-biome gap in understanding of crop 828 and ecosystem responses to elevated CO 2. Current Opinion in Plant Biology (2012). doi:10.1016/j.pbi.2012.01.009

25. Toreti, A. et al. Narrowing uncertainties in the effects of elevated CO2 on crops. Nat. Food 1, 775-782 (2020).

26. Hausfather, Z. \& Peters, G. P. Emissions - the 'business as usual' story is misleading. Nature 577, 618-620 (2020).

27. Minoli, S. et al. Global Response Patterns of Major Rainfed Crops to Adaptation by Maintaining Current Growing Periods and Irrigation. Earth's Futur. 7, 1464-1480 (2019).

28. Wang, X. et al. Emergent constraint on crop yield response to warmer temperature from field experiments. Nat. Sustain. 3, 908-916 (2020).

29. Asseng, S. et al. Rising temperatures reduce global wheat production. Nat. Clim. Chang. 5, 143147 (2014).

30. Kimball, B. A. Crop responses to elevated $\mathrm{CO} 2$ and interactions with $\mathrm{H} 2 \mathrm{O}, \mathrm{N}$, and temperature. Current Opinion in Plant Biology (2016). doi:10.1016/j.pbi.2016.03.006

31. Zabel, F. et al. Large potential for crop production adaptation depends on available future varieties. Glob. Chang. Biol. gcb.15649 (2021). doi:10.1111/gcb.15649

32. Ray, D. K. et al. Climate change has likely already affected global food production. PLoS One 14, 1-18 (2019).

33. Lobell, D. B., Schlenker, W. \& Costa-Roberts, J. Climate trends and global crop production since 1980. Science 333, 616-20 (2011).

34. Ahmad, S. et al. Climate warming and management impact on the change of phenology of the 850 rice-wheat cropping system in Punjab, Pakistan. F. Crop. Res. 230, 46-61 (2019).

35. Porter, J. R. et al. Food security and food production systems. in Climate Change 2014: Impacts, 

Group II to the Fifth Assessment Report of the Intergovernmental Panel on Climate Change (eds. Field, C. B. et al.) 485-533 (Cambridge University Press, 2014).

36. Levis, S., Badger, A., Drewniak, B., Nevison, C. \& Ren, X. CLMcrop yields and water requirements: avoided impacts by choosing RCP 4.5 over 8.5. Clim. Change 146, 501-515 (2018).

37. Falconnier, G. N. et al. Modelling climate change impacts on maize yields under low nitrogen input conditions in sub-Saharan Africa. Glob. Chang. Biol. 26, 5942-5964 (2020).

38. O'Neill, B. C. et al. IPCC reasons for concern regarding climate change risks. Nat. Clim. Chang. 7, 28-37 (2017).

39. Li, Y., Guan, K., Schnitkey, G. D., DeLucia, E. \& Peng, B. Excessive rainfall leads to maize yield loss of a comparable magnitude to extreme drought in the United States. Glob. Chang. Biol. 25, 2325-2337 (2019).

40. Zhu, P., Zhuang, Q., Archontoulis, S. V., Bernacchi, C. \& Müller, C. Dissecting the nonlinear response of maize yield to high temperature stress with model-data integration. Glob. Chang. Biol. 25, 2470-2484 (2019).

42. lizumi, T. et al. Responses of crop yield growth to global temperature and socioeconomic changes. Sci. Rep. 7, 1-10 (2017).

43. Sherwood, S. C. et al. An Assessment of Earth's Climate Sensitivity Using Multiple Lines of Evidence. Rev. Geophys. 58, 1-92 (2020).

44. Flynn, C. M. \& Mauritsen, T. On the climate sensitivity and historical warming evolution in recent coupled model ensembles. Atmos. Chem. Phys. 20, 7829-7842 (2020).

45. Zelinka, M. D. et al. Causes of Higher Climate Sensitivity in CMIP6 Models. Geophys. Res. Lett. 47, 1-12 (2020).

46. Tokarska, K. B. et al. Past warming trend constrains future warming in CMIP6 models. Sci. Adv. 6, 1-14 (2020).

47. Williams, K. D., Hewitt, A. J. \& Bodas-Salcedo, A. Use of Short-Range Forecasts to Evaluate Fast Physics Processes Relevant for Climate Sensitivity. J. Adv. Model. Earth Syst. 12, 1-9 (2020). performance and independence. Earth Syst. Dyn. 11, 995-1012 (2020). 
49. Nijsse, F. J. M. M., Cox, P. M. \& Williamson, M. S. Emergent constraints on transient climate response (TCR) and equilibrium climate sensitivity (ECS) from historical warming in CMIP5 and CMIP6 models. Earth Syst. Dyn. 11, 737-750 (2020).

50. Ridder, N. N., Pitman, A. J. \& Ukkola, A. M. Do CMIP6 Climate Models simulate Global or Regional Compound Events skilfully? Geophys. Res. Lett. 1-11 (2020). doi:10.1029/2020gl091152

51. Fan, X., Miao, C., Duan, Q., Shen, C. \& Wu, Y. The Performance of CMIP6 Versus CMIP5 in Simulating Temperature Extremes Over the Global Land Surface. J. Geophys. Res. Atmos. 125, 1-16 (2020).

52. Xin, X., Wu, T., Zhang, J., Yao, J. \& Fang, Y. Comparison of $<\operatorname{scp}>C M I P 6</ s c p>$ and $<\mathrm{scp}>\mathrm{CMIP} 5</ \mathrm{scp}>$ simulations of precipitation in China and the East Asian summer monsoon. Int. J. Climatol. 40, 6423-6440 (2020).

53. Meinshausen, M. et al. The shared socio-economic pathway (SSP) greenhouse gas concentrations and their extensions to 2500. Geosci. Model Dev. 13, 3571-3605 (2020).

54. Von Bloh, W. et al. Implementing the nitrogen cycle into the dynamic global vegetation, hydrology, and crop growth model LPJmL (version 5.0). Geosci. Model Dev. 11, 2789-2812 (2018).

56. Müller, C. et al. Exploring uncertainties in global crop yield projections in a large ensemble of

57. Franke, J. A. et al. The GGCMI Phase 2 emulators: global gridded crop model responses to

55. Jägermeyr, J. \& Frieler, K. Spatial variations in crop growing seasons pivotal to reproduce global changes in CO2, temperature, water, and nitrogen (version 1.0). Geosci. Model Dev. 13, 23152336 (2020).

58. Allen, L. H. et al. Fluctuations of $\mathrm{CO} 2$ in Free-Air CO2 Enrichment (FACE) depress plant photosynthesis, growth, and yield. Agric. For. Meteorol. 284, (2020).

59. Durand, J. L. et al. How accurately do maize crop models simulate the interactions of atmospheric $\mathrm{CO} 2$ concentration levels with limited water supply on water use and yield? Eur. J. Agron. (2018). doi:10.1016/j.eja.2017.01.002

60. Myers, S. S. et al. Increasing CO2 threatens human nutrition. Nature 510, 139-42 (2014).

61. Zhu, C. et al. Carbon dioxide (CO2) levels this century will alter the protein, micronutrients, and vitamin content of rice grains with potential health consequences for the poorest rice-dependent countries. Sci. Adv. 4, eaaq1012 (2018).

62. Köhler, I. H., Huber, S. C., Bernacchi, C. J. \& Baxter, I. R. Increased temperatures may safeguard the nutritional quality of crops under future elevated $\mathrm{CO} 2$ concentrations. Plant J. 97, 872-886 (2019). 
63. Rising, J. \& Devineni, N. Crop switching reduces agricultural losses from climate change in the United States by half under RCP 8.5. Nat. Commun. 11, 4991 (2020).

64. Asseng, S. et al. Climate change impact and adaptation for wheat protein. Glob. Chang. Biol. 25, 155-173 (2019).

65. Hawkins, E. \& Sutton, R. The potential to narrow uncertainty in regional climate predictions. Bull. Am. Meteorol. Soc. 90, 1095-1107 (2009).

66. Giorgi, F. \& Bi, X. Time of emergence (TOE) of GHG-forced precipitation change hot-spots. Geophys. Res. Lett. 36, L06709 (2009).

67. Lange, S. WFDE5 over land merged with ERA5 over the ocean (W5E5). V. 1.0. GFZ Data Services (2019). doi:https://doi.org/10.5880/pik.2019.023

68. Cucchi, M. et al. WFDE5: Bias-adjusted ERA5 reanalysis data for impact studies. Earth Syst. Sci. Data 12, 2097-2120 (2020).

69. Ruane, A. C. et al. Strong regional influence of climatic forcing datasets on global crop model ensembles. Agric. For. Meteorol. 300, 108313 (2021).

70. FAO. FAOSTAT, United Nation's Food and Agricultural Organization, Rome. (2019). Available at: http://www.fao.org/faostat/. (Accessed: 10th July 2019)

71. Portmann, F. T., Siebert, S. \& Döll, P. MIRCA2000 - Global monthly irrigated and rainfed crop areas around the year 2000: A new high-resolution data set for agricultural and hydrological modeling. Global Biogeochem. Cycles 24, 1-24 (2010).

72. Siebert, S. et al. A global data set of the extent of irrigated land from 1900 to 2005. Hydrol. Earth Syst. Sci. 19, 1521-1545 (2015).

73. Mueller, N. D. et al. Closing yield gaps through nutrient and water management. Nature 490, 254-7 (2012).

74. Hurtt, G. C. et al. Harmonization of global land use change and management for the period 8502100 (LUH2) for CMIP6. Geosci. Model Dev. 13, 5425-5464 (2020).

75. Zhang, B. et al. Global manure nitrogen production and application in cropland during 18602014: A 5 arcmin gridded global dataset for Earth system modeling. Earth Syst. Sci. Data 9, 667-678 (2017).

76. Tian, H. et al. The Global N2O Model Intercomparison Project. Bull. Am. Meteorol. Soc. 99, 1231-1251 (2018).

77. Nachtergaele, F. et al. Harmonized World Soil Database (version 1.2). FAO, Rome, Italy IIASA,

78. Shangguan, W., Dai, Y., Duan, Q., Liu, B. \& Yuan, H. A global soil data set for earth system modeling. J. Adv. Model. Earth Syst. 6, 249-263 (2014). 9, (2014). 
80. Elliott, J. et al. The Global Gridded Crop Model Intercomparison: data and modeling protocols for Phase 1 (v1.0). Geosci. Model Dev. 8, 261-277 (2015).

81. Müller, C. et al. Global gridded crop model evaluation: benchmarking, skills, deficiencies and implications. Geosci. Model Dev. 10, 1403-1422 (2017).

963

82. Franke, J. A. et al. The GGCMI Phase 2 experiment: global gridded crop model simulations under uniform changes in $\mathrm{CO} 2$, temperature, water, and nitrogen levels (protocol version 1.0). Geosci. Model Dev. 13, 2315-2336 (2020).

83. Ruane, A. C. et al. Multi-wheat-model ensemble responses to interannual climate variability. Environ. Model. Softw. 81, 86-101 (2016).

84. Wang, R., Bowling, L. C. \& Cherkauer, K. A. Estimation of the effects of climate variability on crop yield in the Midwest USA. Agric. For. Meteorol. 216, 141-156 (2016).

85. Folberth, C., Gaiser, T., Abbaspour, K. C., Schulin, R. \& Yang, H. Regionalization of a largescale crop growth model for sub-Saharan Africa: Model setup, evaluation, and estimation of maize yields. Agric. Ecosyst. Environ. 151, 21-33 (2012).

86. International Food Policy Research Institute. Global Spatially-Disaggregated Crop Production Statistics Data for 2010 Version 1.0. Harvard Dataverse V1 (2019). Available at: https://doi.org/10.7910/DVN/PRFF8V. (Accessed: 15th February 2019)

87. Jägermeyr, J. et al. A regional nuclear conflict would compromise global food security. Proc. Natl. Acad. Sci. 117, 7071-7081 (2020).

88. International Food Policy Research Institute (IFPRI) \& International Institute for Applied Systems Analysis (IIASA). Global Spatially-Disaggregated Crop Production Statistics Data for 2005 Version 3.2. Harvard Dataverse V9 (2016). Available at: https://doi.org/10.7910/DVN/DHXBJX. (Accessed: 15th February 2019)

984 90. Reynolds, M. \& Braun, H. Benefits to low-input agriculture. Nat. Plants 5, 652-653 (2019).

985 91. Kottek, M., Grieser, J., Beck, C., Rudolf, B. \& Rubel, F. World Map of the Köppen-Geiger climate 986 classification updated. Meteorol. Zeitschrift 15, 259-263 (2006).

987 92. John P. Snyder \& Voxland, P. M. An album of map projections. (1989). doi:10.3133/pp1453 988 


\section{Supplementary Files}

This is a list of supplementary files associated with this preprint. Click to download.

- SupplementJaegermeyretalNATFOOD20111649T.pdf 